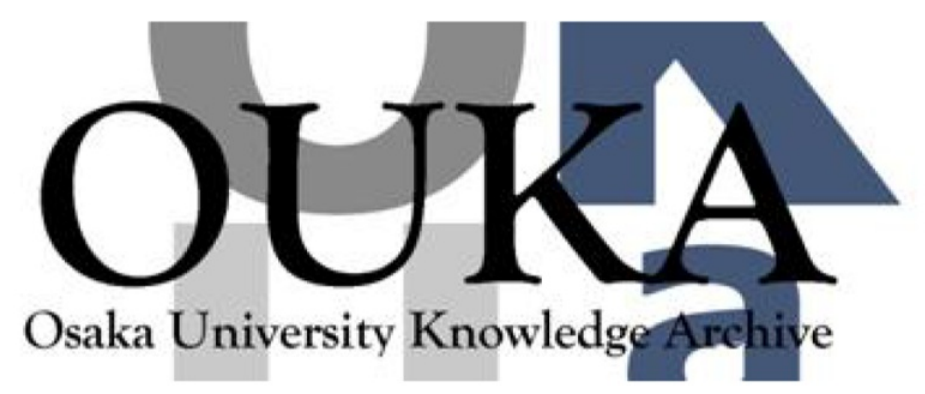

\begin{tabular}{|c|l|}
\hline Title & $\begin{array}{l}\text { A Transformer Noise-Canceling ULtra-Wideband } \\
\text { CMOS Low-Noise Amplifier }\end{array}$ \\
\hline Author(s) & $\begin{array}{l}\text { Kihara, Takao; Matsuoka, Toshimasa; Taniguchi, } \\
\text { Kenj i }\end{array}$ \\
\hline Citation & $\begin{array}{l}\text { IEICE Transactions on Electronics. E93-C(2) } \\
\text { p. 187-p. 199 }\end{array}$ \\
\hline Issue Date & $2010-02$ \\
\hline oaire:version & VoR \\
\hline URL & https://hdl.handle.net/11094/51722 \\
\hline rights & copyright $\odot 2010$ IEICE \\
\hline Note & \\
\hline
\end{tabular}

Osaka University Knowledge Archive : OUKA

https://ir. Library. osaka-u. ac. jp/

Osaka University 


\section{A Transformer Noise-Canceling Ultra-Wideband CMOS Low-Noise Amplifier}

Takao KIHARA $^{\dagger \text { a) }}$, Nonmember, Toshimasa MATSUOKA ${ }^{\dagger}$, and Kenji TANIGUCHI $^{\dagger}$, Members $^{\circ}$

SUMMARY Previously reported wideband CMOS low-noise amplifiers (LNAs) have difficulty in achieving both wideband input impedance matching and low noise performance at low power consumption and low supply voltage. We present a transformer noise-canceling wideband CMOS LNA based on a common-gate topology. The transformer, composed of the input and shunt-peaking inductors, partly cancels the noise originating from the common-gate transistor and load resistor. The combination of the transformer with an output series inductor provides wideband input impedance matching. The LNA designed for ultra-wideband (UWB) applications is implemented in a $90 \mathrm{~nm}$ digital CMOS process. It occupies $0.12 \mathrm{~mm}^{2}$ and achieves $\left|S_{11}\right|<-10 \mathrm{~dB}, N F<4.4 \mathrm{~dB}$, and $\left|S_{21}\right|>9.3 \mathrm{~dB}$ across $3.1-10.6 \mathrm{GHz}$ with a power consumption of $2.5 \mathrm{~mW}$ from a $1.0 \mathrm{~V}$ supply. These results show that the proposed topology is the most suitable for low-power and low-voltage UWB CMOS LNAs.

key words: CMOS, low-noise amplifier (LNA), noise cancellation, ultrawideband $(U W B)$, transformer

\section{Introduction}

Ultra-wideband (UWB) technology has attracted much interest in recent years, because of its ability to realize highspeed wireless personal area networks (WPANs), in which electronic devices are required to transfer large amounts of data, such as audio or video files, at a high data transfer rate. UWB frequency bands assigned from 3.1 to $10.6 \mathrm{GHz}$ are utilized by two different communication systems: multiband orthogonal frequency division multiplexing (MB-OFDM) UWB [1] or single-carrier direct sequence (DS) UWB [2]. The MB-OFDM UWB system using 14 sub-bands, each with a bandwidth of $528 \mathrm{MHz}$, transmits signals modulated by OFDM in the subband. The data rate is up to $480 \mathrm{Mbps}$. The DS-UWB system spreads the spectrum over the low band $(3.1-4.85 \mathrm{GHz})$ or high band $(6.2-9.7 \mathrm{GHz})$, and provides a maximum data rate of $1320 \mathrm{Mbps}$. In either case, wideband low-noise amplifiers (LNAs) are essential for the RF front-ends of UWB receivers.

The UWB LNA must meet several stringent requirements: input impedance matching, low noise performance, and sufficient gain across $3.1-10.6 \mathrm{GHz}$ at low power consumption, a low supply voltage, and low cost (i.e., small area and requiring no additional layers). In addition, it is desirable to implement the LNA with digital CMOS technologies for the integration of RF front-ends and digital circuits. Al-

\footnotetext{
Manuscript received May 2, 2009.

Manuscript revised August 30, 2009.

${ }^{\dagger}$ The authors are with the Division of Electrical, Electronics and Information Engineering, Graduate School of Engineering, Osaka University, Suita-shi, 565-0871 Japan.

a)E-mail: kihara@si.eei.eng.osaka-u.ac.jp DOI: 10.1587/transele.E93.C.187
}

though several wideband CMOS LNAs have been proposed in recent years, none of them have simultaneously met all these requirements. An LNA with wideband LC matching networks [3] consumes a large chip area. Although resistive-feedback LNAs [4]-[6] and common-drain feedback LNAs [7], [8] occupy small chip areas, they require high power consumption and high supply voltages to simultaneously achieve wideband input impedance matching and low noise performance. A reactive-feedback LNA [9], [10] demands two thick metal layers to form a transformer that provides a reactive feedback. Noise-canceling LNAs [11]-[14] require additional circuits and power consumption. Distributed LNAs [15], [16] consume much higher power and larger areas than other LNAs.

In this paper, we propose a transformer noise-canceling common-gate LNA employing an output series inductor [17]. The proposed LNA is suitable for low-power and low-voltage operation, and achieves $\left|S_{11}\right|<-10 \mathrm{~dB}, N F<$ $4.4 \mathrm{~dB}$, and $\left|S_{21}\right|>9.3 \mathrm{~dB}$ across $3.1-10.6 \mathrm{GHz}$. This paper is organized as follows. Section 2 describes the proposed circuit topology and noise cancellation mechanisms. The noise, input admittance, gain, stability, and group delay of the proposed LNA are analyzed in Sect.3. Section 4 describes the design methodology for the LNA. Section 5 shows the measurements of the LNA implemented in a $90 \mathrm{~nm}$ digital CMOS process, and Sect. 6 concludes the paper.

\section{Transformer Noise-Canceling LNA}

This section presents the circuit topology of the transformer noise-canceling CMOS LNA and the noise cancellation mechanisms.

\subsection{Circuit Topology}

Figure 1 shows a schematic of the proposed LNA, based on a common-gate (CG) LNA with a shunt-peaking inductor. The CG topology is suitable for low-voltage operation, because it does not require a cascode transistor to alleviate the Miller effect from the CG transistor $M_{1}$ [18]. The main difference between the proposed LNA and the CG LNA is that the input and shunt-peaking inductors, $L_{p}$ and $L_{s}$, are magnetically coupled to form a transformer. A similar topology has been reported for narrowband applications [19]. The transformer cancels the noise produced by $M_{1}$ and the load resistor $R_{L}$, thereby improving the noise performance with- 


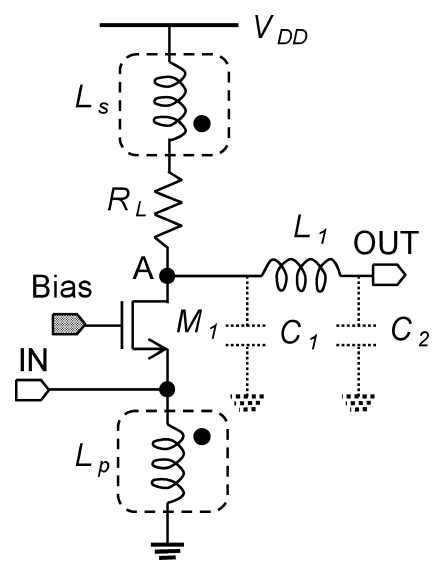

Fig. 1 Schematic of the proposed LNA.

out additional circuits or increased power consumption. The transformer also provides a positive feedback, whose mechanism is as follows: An output current generated by a signal voltage flows through $L_{s}$, which induces a voltage that is in phase with the signal voltage to $L_{p}$. The output series inductor $L_{1}$ forms a $\pi$ network with the parasitic capacitances, $C_{1}$ and $C_{2}$, extending not only the gain bandwidth, but also the input bandwidth, which is defined as the frequency range of $\left|S_{11}\right|<-10 \mathrm{~dB}$. The chip area of the proposed LNA is the same as that of a CG LNA with a shunt-peaking inductor, because $L_{p}$ can be stacked on $L_{s}$, i.e., a stacked transformer, which occupies the area of one inductor.

\subsection{Noise Cancellation}

The transformer partly cancels the output noise originating from the CG transistor $M_{1}$ and load resistor $R_{L}$, thereby improving the LNA noise performance. The small-signal circuit of the proposed LNA is shown in Fig. 2, where the voltage supply terminal $\left(V_{\mathrm{DD}}\right)$ is connected to the AC ground; the noise of the signal source resistance $R_{s}, M_{1}$, and $R_{L}$ are represented by the noise current sources $i_{n s}, i_{n d}$, and noise voltage source $v_{n R_{L}}$, respectively; $M$, given by $k \sqrt{L_{p} L_{s}}$, is the mutual inductance of the transformer and $k$ the magnetic coupling factor; $C_{p}$ represents the sum of the gate-source capacitance of $M_{1}$ and the parasitic capacitances of the input pad and $L_{p} ; Z_{L}$ is the load impedance considering the right hand side of output node A. The mechanisms for the noise cancellation are conceptually illustrated in Figs. 3(a) and (b). The transformer detects noise currents flowing through the primary (or secondary) inductor $L_{p}$, inducing voltages correlated with the currents to the secondary (or primary) inductor $L_{s}$.

\subsubsection{Transistor Noise Cancellation}

The output noise voltage generated by $i_{n d}$ is partly canceled by the induced noise voltage originating from $i_{n d}$ flowing through $L_{p}$, as shown in Fig. 3(a). The noise current $i_{n d}$ first flowing through $L_{s}$ and $R_{L}$ generates a noise voltage $v_{n 1}=$

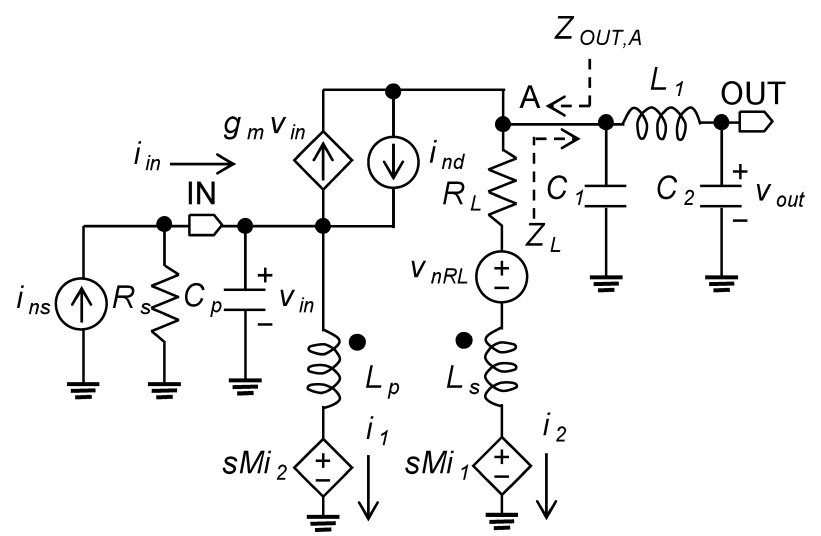

Fig. 2 Small-signal equivalent circuit with noise sources.

$-i_{n d}\left(R_{L}+s L_{s}\right)$, and then a noise voltage $v_{n 1}^{\prime}=-i_{n d} \cdot s M$ is induced in $L_{p}$. Next, $i_{n d}$ flows through $L_{p}$, producing a noise voltage $v_{n 2}=i_{n d} \cdot s L_{p}$, which is canceled by $v_{n 1}^{\prime}$. Here, the transformer induces a noise voltage $v_{n 2}^{\prime}=i_{n d} \cdot s M$ in $L_{s}$. The induced noise voltage $v_{n 2}^{\prime}$ is correlated and in antiphase with $v_{n 1}$ and hence the total output noise voltage is reduced: $-i_{n d}\left(R_{L}+s L_{s}-s M\right)$. The expression of the output noise voltage (at node A) including the effect of $Z_{L}$ can be derived from Fig. 2:

$$
\begin{aligned}
& v_{\text {out } i_{\text {nd }}}=-i_{n d} Z_{L} \\
& \times \frac{n^{2} k^{2}-n k+\left(\frac{1}{R_{s}}+s C_{p}+\frac{1}{s L_{p}}\right)\left(R_{L}+s\left(1-k^{2}\right) L_{s}\right)}{\left(\frac{1}{R_{s}}+Y_{I N}\right)\left(Z_{L}+R_{L}+s\left(1-k^{2}\right) L_{s}\right)},
\end{aligned}
$$

where $n=\sqrt{L_{s} / L_{p}}$ is the turn ratio of the transformer; $Y_{I N}=$ $i_{\text {in }} / v_{\text {in }}$, described in the next section, is the input admittance of the LNA, and $i_{\text {in }}$ and $v_{\text {in }}$ are the input current and voltage, respectively, as shown in Fig. 2. The term of $s\left(1-k^{2}\right) L_{s}$ in the numerator of Eq. (1) shows that the transistor noise is partly canceled by the transformer.

\subsubsection{Load Resistor Noise Cancellation}

The CG transistor $M_{1}$ drains a part of the output noise current originating from $v_{n R_{L}}$, reducing the output noise voltage, as shown in Fig. 3(b). The noise current due to $v_{n R_{L}}$, which is given by $v_{n R_{L}} /\left(s L_{s}+R_{L}+Z_{L}\right)$, first flows through $L_{s}$ and then the transformer induces a noise voltage $v_{n R_{L}}^{\prime}=$ $-s M v_{n R_{L}} /\left(s L_{s}+R_{L}+Z_{L}\right)$ in $L_{p}$. The transistor $M_{1}$ detects a gate-source voltage $v_{g s}=v_{n R_{L}}^{\prime} / s L_{p}\left(1 / R_{s}+g_{m}+s C_{p}+1 / s L_{p}\right)$, and drains noise current of $g_{m} v_{g s}$ accordingly. This results in a reduction of the output noise current originating from $v_{n R_{L}}$. Considering the noise current due to $M_{1}$, we can obtain the output noise current flowing $Z_{L}$ :

$$
i_{\text {out }, v_{n R_{L}}}=\frac{\left(\frac{1}{R_{s}}+(1-n k) g_{m}+s C_{p}+\frac{1}{s L_{p}}\right) v_{n R_{L}}}{\left(\frac{1}{R_{s}}+Y_{I N}\right)\left(Z_{L}+R_{L}+s\left(1-k^{2}\right) L_{s}\right)},
$$

and the output noise voltage (at node A), $v_{\text {out } v_{n R_{L}}}$, is given by $i_{\text {out }, v_{n R_{L}}} Z_{L}$. The term $-n k g_{m}$ in Eq. (2) originates from the 


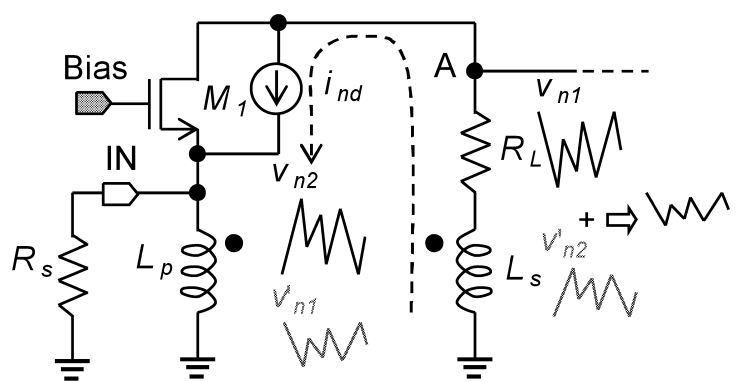

(a)

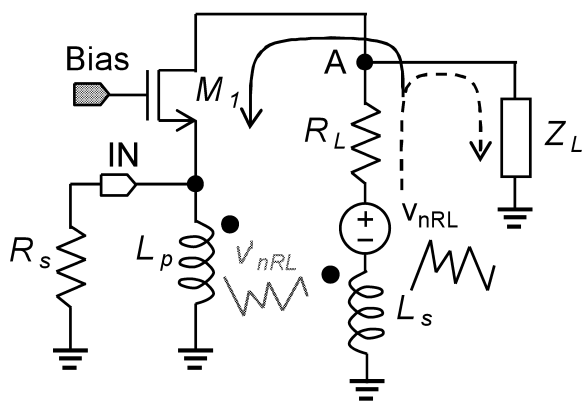

(b)

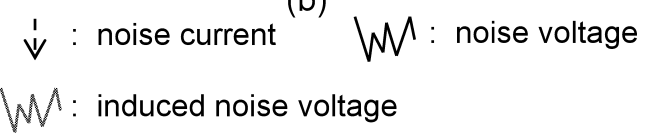

Fig. 3 Mechanisms for noise cancellation of (a) $i_{n d}$ and (b) $v_{n R_{L}}$.

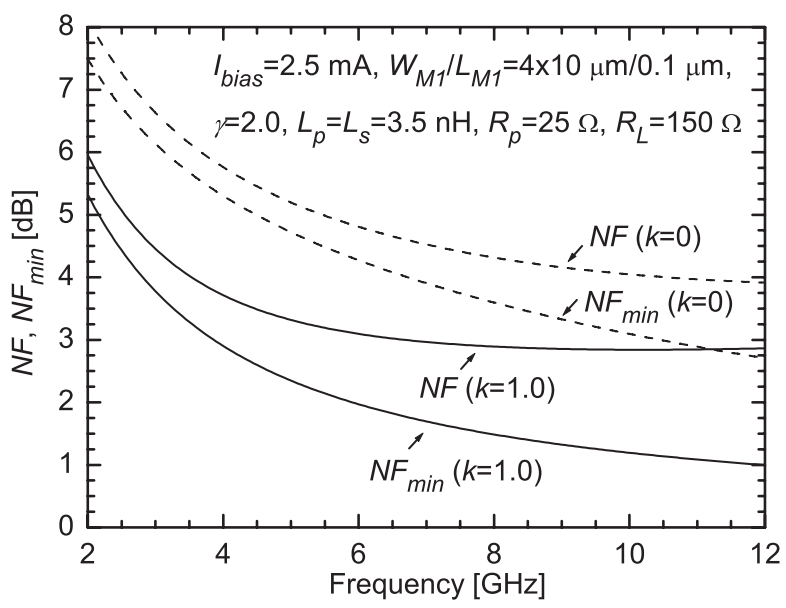

Fig. 4 Simulated $N F$ and $N F_{\min }$ of the LNAs with and without noise cancellation $(k=0,1.0)$.

noise cancellation.

\subsubsection{Verification}

The effectiveness of the transformer noise cancellation is verified through simulation. Figure 4 shows the simulated $N F$ and $N F_{\min }$ of the proposed LNAs with and without the noise cancellation (i.e., $k=0,1.0$ ), where $90 \mathrm{~nm}$ CMOS process parameters are used and $R_{p}$ represents the parasitic resistance of $L_{p}$. The LNA with $k=0$ corresponds to a CG LNA with a load resistor and shunt-peaking inductor. The

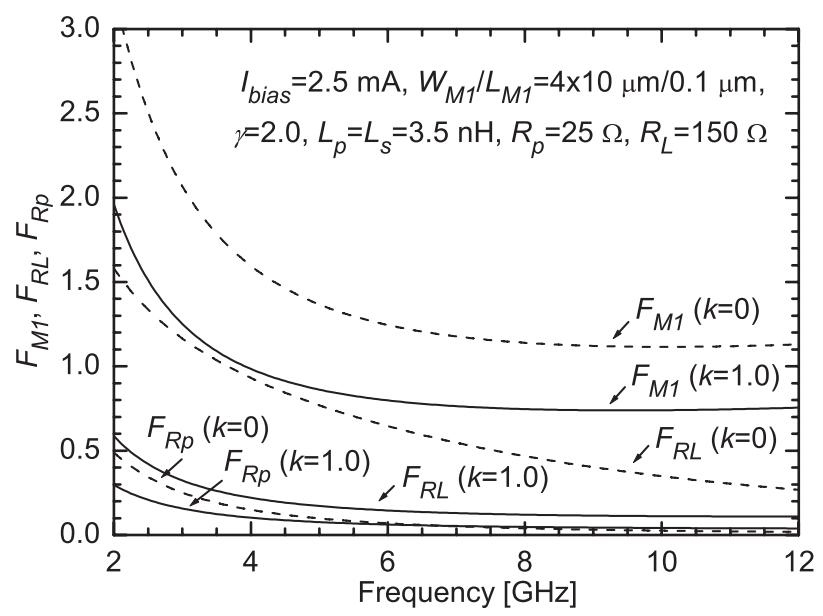

Fig. 5 Simulated noise contributions from $M_{1}, R_{L}$, and $R_{p}$ to the LNAs with and without noise cancellation $(k=0,1.0)$.

$N F$ of the LNA with $k=1.0$ is up to $2.2 \mathrm{~dB}$ lower than that of the LNA with $k=0$. Figure 5 shows the simulated noise factors contributed from $M_{1}, R_{L}$, and $R_{p}\left(F_{M_{1}}, F_{R_{L}}\right.$, and $F_{R_{p}}$, respectively) with and without the noise cancellation. The transformer reduces $F_{M_{1}}$ by up to $35 \%$ and $F_{R_{L}}$ by $65 \%$. The contribution from $R_{p}$ also slightly decreases and hence $R_{p}$ contributes little to the overall $N F$ (i.e., $0.1 \mathrm{~dB}$ in Fig. 4). The noise contributions from $M_{1}$ and $R_{L}$ change with the turn ratio. A noise optimization procedure will be presented in the next section.

\section{Circuit Analysis}

The transformer improves the LNA noise performance at the cost of the input and gain bandwidths. The output series inductor $L_{1}$ extends both the bandwidths. In this section, the noise, input admittance, gain, stability, and group delay of the LNA are analyzed, and noise optimization and impedance matching procedures are presented.

\subsection{Noise}

The amount of noise cancellation is mainly determined by the turn ratio of the transformer. From the small-signal equivalent circuit shown in Fig. 2, the output noise voltage due to $R_{S}$ is given by

$$
v_{\text {out }, i_{n s}}=Z_{L} \frac{\left(g_{m} R_{L}+n k+s\left(1-k^{2}\right) L_{s} g_{m}\right) i_{n s}}{\left(\frac{1}{R_{s}}+Y_{I N}\right)\left(Z_{L}+R_{L}+s\left(1-k^{2}\right) L_{s}\right)} .
$$

Using Eqs. (1)-(3), we obtain the noise factor of the proposed LNA:

$$
\begin{aligned}
& F \approx 1+F_{M_{1}}+F_{R_{L}}, \\
& F_{M_{1}}=\left|\frac{v_{\text {out }, i_{n d}}}{v_{\text {out }, i_{n s}}}\right|^{2}
\end{aligned}
$$




$$
\begin{aligned}
& =\left|\frac{n^{2}-n+\left(\frac{1}{R_{s}}+j \omega C_{p}+\frac{1}{j \omega L_{p}}\right) R_{L}}{g_{m} R_{L}+n}\right|^{2} \gamma g_{d 0} R_{s}, \\
F_{R_{L}} & =\left|\frac{v_{\text {out }, v_{n R_{L}}}}{v_{\text {out } i_{n s}}}\right|^{2} \\
& =\left|\frac{(1-n) g_{m} R_{L}+\left(\frac{1}{R_{s}}+j \omega C_{p}+\frac{1}{j \omega L_{p}}\right) R_{L}}{g_{m} R_{L}+n}\right|^{2} \frac{R_{s}}{R_{L}},
\end{aligned}
$$

where $g_{d 0}$ is the zero-bias drain conductance of $M_{1}$; the coefficient of channel thermal noise $\gamma$ equals $2 / 3$ in longchannel MOSFETs, but exceeds this value in short-channel MOSFETs [20]-[22]. The value of $\gamma$ in a fabricated $90 \mathrm{~nm}$ MOSFET is approximately two, shown by the measured and simulated $N F$ of the LNA, as will be shown in Sect. 5. For simplicity, the magnetic coupling factor $k$ is assumed to be one. The parasitic resistance of $L_{p}$, the parasitic capacitance between $L_{p}$ and $L_{s}$, and the induced-gate noise current of $M_{1}$ are ignored, because they do not have a significant effect on the overall $N F$. The transconductance $g_{m}$ and load resistance $R_{L}$ cannot be optimized for noise, because they are determined from input impedance matching conditions, as will be shown in the following subsection.

The optimum $n$ for the noise performance can be obtained from Eqs. (4)-(6). Setting the derivatives of Eqs. (5) and (6) with respect to $n$ to zero (i.e., $\partial F_{M_{1}} / \partial n=0$ and $\partial F_{R_{L}} / \partial n=0$ for $\omega=1 / \sqrt{L_{p} C_{p}}$ ), we can obtain

$$
\begin{aligned}
& n_{o p t, i_{n d}}=-g_{m} R_{L}+\sqrt{\left(g_{m} R_{L}\right)^{2}+g_{m} R_{L}+\frac{R_{L}}{R_{s}}}, \\
& n_{o p t, v_{n R_{L}}}=1+\frac{1}{g_{m} R_{s}},
\end{aligned}
$$

for which a minimum $F_{M_{1}}$ and $F_{R_{L}}$ are achieved, respectively. Similarly, the optimum $n$ for $F, n_{\text {opt }}$, can be obtained from $\partial F / \partial n=0$ :

$$
\begin{aligned}
& \left(n_{\text {opt }}^{2}-n_{\text {opt }}+\frac{R_{L}}{R_{s}}\right) \\
& \times\left(n_{\text {opt }}^{2}+\left(2 n_{\text {opt }}-1\right) g_{m} R_{L}-\frac{R_{L}}{R_{s}}\right) \gamma g_{d 0} \\
& -\left(\left(1-n_{\text {opt }}\right) g_{m}+\frac{1}{R_{s}}\right)\left(g_{m}^{2} R_{L}+g_{m}+\frac{1}{R_{s}}\right) R_{L}=0 .
\end{aligned}
$$

Figures 6(a) and (b) show the calculated $F, F_{M_{1}}$, and $F_{R_{L}}$ $\left(R_{L}=50 \Omega\right)$ versus $n$ and $N F$ with $R_{L}$ as a parameter at $\omega=1 / \sqrt{L_{p} C_{p}}$, respectively. For $R_{L}=50 \Omega$, minimum $F_{M_{1}}$, $F_{R_{L}}$, and $F$ are achieved for $n$ of $0.68,1.66$, and 1.0, given by Eqs. (7)-(9), respectively. Figure 6(b) shows that the calculated $N F\left(R_{L}=150 \Omega\right)$ for $n=1.0$ is consistent with the simulated $N F(k=1.0)$ at $7.2 \mathrm{GHz}$, shown in Fig. 4, although $R_{p}$ is ignored in Eqs. (4)-(6). Moreover, the $N F$ becomes a minimum around one even with varying $R_{L}$ from 50 to $200 \Omega$. A large $n$ makes the LNA unstable, as will be

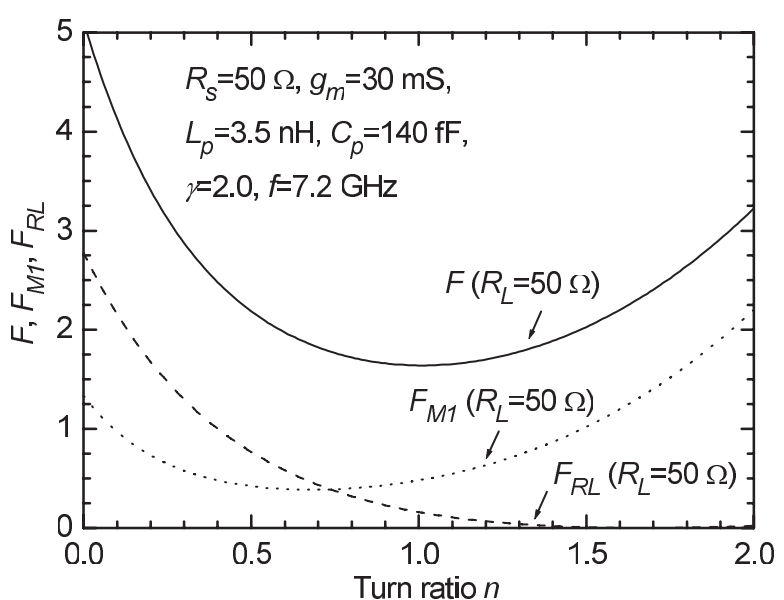

(a)

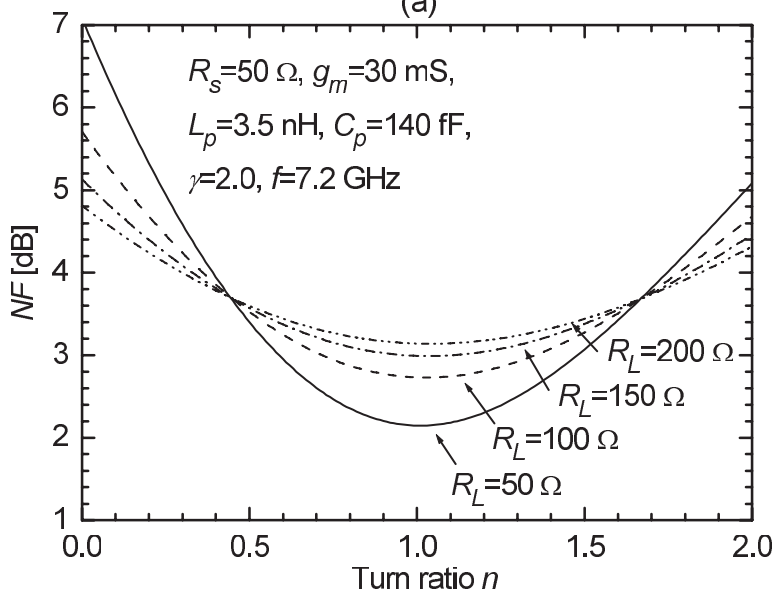

(b)

Fig. 6 Calculated (a) $F, F_{M_{1}}$, and $F_{R_{L}}\left(R_{L}=50 \Omega\right.$ ) versus $n$ and (b) $N F$ with $R_{L}$ as a parameter.

explained in Section 3.4, and leads to an increase in the parasitic capacitance of $L_{s}$, causing poor high-frequency performance. The optimum $n$ is thus determined to be one.

\subsection{Input Impedance Matching}

In the proposed topology with the input and shunt-peaking inductors coupled, the output load affects the LNA input impedance through the coupling. The output series inductor $L_{1}$ contributes to wideband input impedance matching. From Fig. 2, the input admittance of the proposed LNA, $Y_{I N}$, is given by

$$
Y_{I N}(j \omega)=g_{m}+j \omega C_{p}+\frac{1}{j \omega L_{p}}+Y_{T} .
$$

The first three terms in Eq. (10) represent the input admittance of the CG LNA. The last term $Y_{T}$ is generated by coupling $L_{p}$ and $L_{s}$, and is given by

$$
Y_{T}(j \omega)=\frac{n k\left(n k-g_{m} Z_{L}\right)}{R_{L}+Z_{L}+j \omega n^{2} L_{p}\left(1-k^{2}\right)} .
$$

When $L_{1}$ is connected in series with the output, $Z_{L}$ is expressed as 

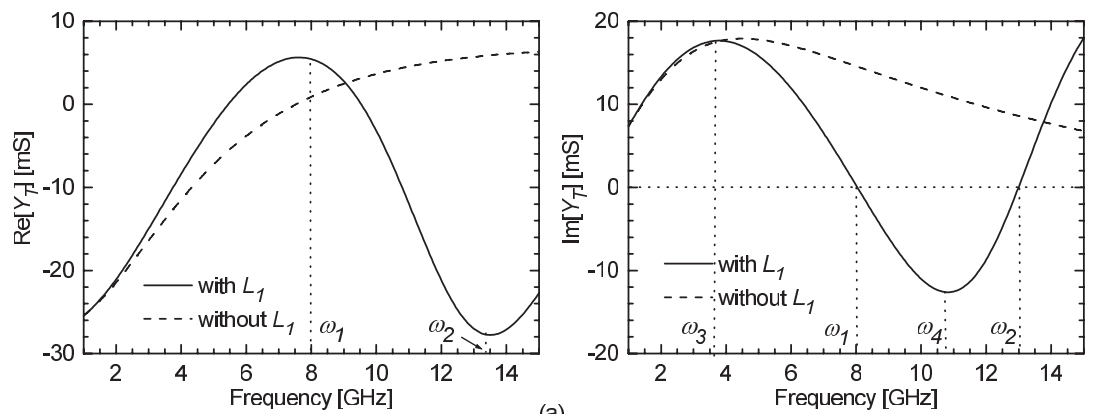

$g_{\mathrm{m}}=30 \mathrm{mS}, C_{D}=140 \mathrm{fF}, L_{p}=3.5 \mathrm{nH}, R_{\mathrm{L}}=150 \Omega$,
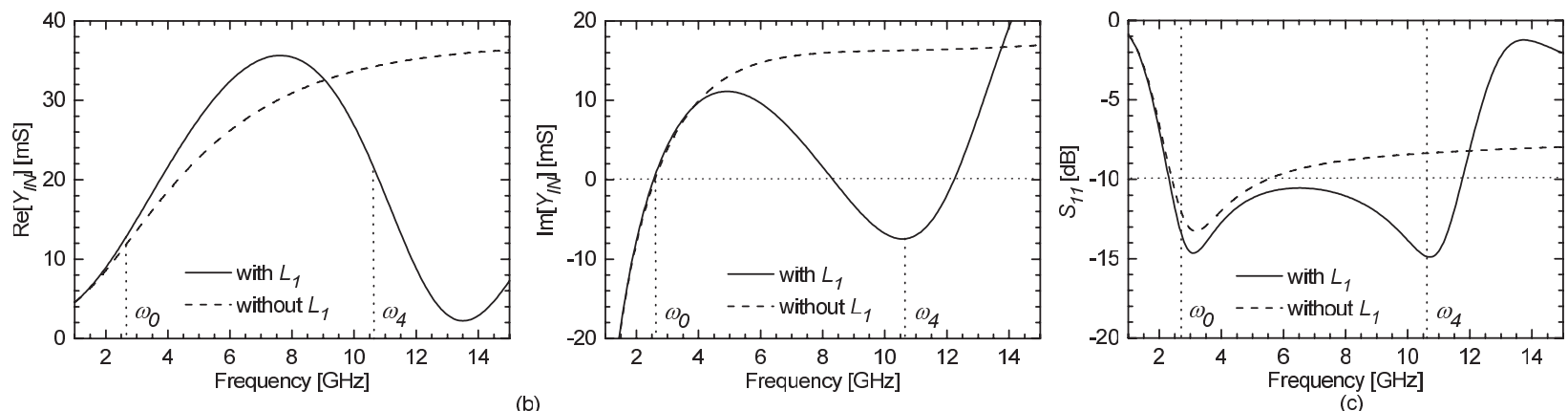

Fig. 7 Calculated real and imaginary parts of (a) $Y_{T}$ and (b) $Y_{I N}$, and (c) $S_{11}$ of the LNAs with and without $L_{1}$.

$$
Z_{L}(j \omega)=\frac{1}{j \omega C_{1}} / /\left(j \omega L_{1}+\frac{1}{j \omega C_{2}}\right),
$$

where $C_{1}$ represents the sum of the gate-drain capacitance of $M_{1}$ and the parasitic capacitance of $L_{1} ; C_{2}$, which is typically larger than $C_{1}$, represents the sum of the input capacitance of the following stage and the parasitic capacitance of $L_{1}$. Equations (10)-(12) show that $Y_{I N}$ is a function of $Y_{T}$, whose frequency behavior significantly depends on that of $Z_{L}$. From Eqs. (10)-(12), the calculated frequency behavior of $Y_{T}$ is shown in Fig. 7(a) (solid line), and that of $Y_{T}$ for $Z_{L}(j \omega)=1 / j \omega\left(C_{1}+C_{2}\right)$ is also shown for comparison (dashed line). The $\pi$ network consisting of $C_{1}, L_{1}$, and $C_{2}$ acts as a short or an open [23] (i.e., $Z_{L}=0$ or $\infty$ ), providing a maximum and minimum $\operatorname{Re}\left[Y_{T}(j \omega)\right]$ and $\operatorname{Im}\left[Y_{T}(j \omega)\right]$ :

$$
\begin{aligned}
\operatorname{Re}\left[Y_{T}(j \omega)\right]_{\max } & \approx \frac{n^{2} k^{2}}{R_{L}}, \\
\operatorname{Re}\left[Y_{T}(j \omega)\right]_{\min } & \approx-n k g_{m}, \\
\operatorname{Im}\left[Y_{T}(j \omega)\right]_{\max } & \approx \frac{n k}{2}\left(g_{m}+\frac{n k}{R_{L}}\right), \\
\operatorname{Im}\left[Y_{T}(j \omega)\right]_{\min } & \approx-\frac{n k}{2} \\
& \times\left(\frac{g_{m} \frac{L_{1} C_{2}}{C_{1}+C_{2}}}{\frac{L_{1} C_{2}}{C_{1}+C_{2}}+n^{2}\left(1-k^{2}\right) L_{p}}+\frac{n k}{R_{L}}\right),
\end{aligned}
$$

at the following frequencies:

$$
\begin{aligned}
& \omega_{1}=\frac{1}{\sqrt{L_{1} C_{2}}}, \\
& \omega_{2}=\frac{1}{\sqrt{L_{1} \frac{C_{1} C_{2}}{C_{1}+C_{2}}}},
\end{aligned}
$$

$$
\begin{aligned}
& \omega_{3} \approx \omega_{c}=\frac{1}{R_{L}\left(C_{1}+C_{2}\right)}, \\
& \omega_{4} \approx \frac{R_{L}}{\frac{L_{1} C_{2}}{C_{1}+C_{2}}+n^{2}\left(1-k^{2}\right) L_{p}},
\end{aligned}
$$

respectively. The above equations and approximations are derived from the following conditions: $Z_{L}=0$ and $j \omega n^{2}\left(1-k^{2}\right) L_{p}$ is ignored against $R_{L}$ in Eqs. (13) and (17); $Z_{L}=\infty$ in Eqs. (14) and (18); $Z_{L}=1 / j \omega\left(C_{1}+C_{2}\right)$ and $\omega^{2} n^{2}\left(1-k^{2}\right) L_{p}\left(C_{1}+C_{2}\right) \ll 1$ in Eqs. (15) and (19); $Z_{L}=j \omega L_{1} C_{2} /\left(C_{1}+C_{2}\right)$ in Eqs. (16) and (20). A negative $\operatorname{Re}\left[Y_{T}(j \omega)\right]$ shown in Eq. (14) originates from the positive feedback provided by the transformer. The calculated $Y_{I N}$ is also shown in Fig. 7(b). The real part of $Y_{I N}, \operatorname{Re}\left[Y_{I N}(j \omega)\right]$, is simply shifted by $g_{m}$ from $\operatorname{Re}\left[Y_{T}(j \omega)\right]$, and the imaginary part of $Y_{I N}, \operatorname{Im}\left[Y_{I N}(j \omega)\right]$, becomes zero at resonance frequencies. The first resonance frequency $\omega_{0}$ is derived from the following equation:

$$
\begin{aligned}
\operatorname{Im}\left[Y_{I N}\left(j \omega_{0}\right)\right] \approx & j \omega_{0} C_{p}+\frac{1}{j \omega_{0} L_{p}} \\
& +j \omega_{0} \frac{n k\left(C_{1}+C_{2}\right)\left(n k+g_{m} R_{L}\right)}{1+\omega_{0}^{2} R_{L}^{2}\left(C_{1}+C_{2}\right)^{2}}=0,
\end{aligned}
$$

where the last term in $\operatorname{Im}\left[Y_{I N}\left(j \omega_{0}\right)\right]$ is $\operatorname{Im}\left[Y_{T}\left(j \omega_{0}\right)\right]$ for $Z_{L}=$ $1 / j \omega_{0}\left(C_{1}+C_{2}\right)$ and $\omega_{0}^{2} n^{2}\left(1-k^{2}\right) L_{p}\left(C_{1}+C_{2}\right) \ll 1$.

The requirements of $g_{m}$ and $R_{L}$ are derived from the input impedance matching condition in the frequency range from $\omega_{0}$ to $\omega_{1}$. For input impedance matching $\left(\left|S_{11}\right|<\right.$ $-10 \mathrm{~dB}), Y_{I N}$ must satisfy the following condition:

$$
\left|S_{11}\right|=\left|\frac{1-R_{S} Y_{I N}}{1+R_{S} Y_{I N}}\right|<0.316 .
$$


When $\operatorname{Im}\left[Y_{I N}(j \omega)\right]=0$, Eq. (22) can be simplified to

$$
10 \mathrm{mS}<\operatorname{Re}\left[Y_{I N}(j \omega)\right]<38 \mathrm{mS} .
$$

At $\omega_{0}, \operatorname{Im}\left[Y_{I N}\left(j \omega_{0}\right)\right]=0$ and the real part of $Y_{I N}(j \omega)$ is approximated as

$$
\operatorname{Re}\left[Y_{I N}\left(j \omega_{0}\right)\right] \approx g_{m}\left(1-\frac{n k}{1+\omega_{0}^{2} R_{L}^{2}\left(C_{1}+C_{2}\right)^{2}}\right),
$$

where $\omega_{0}^{2} n k R_{L}\left(C_{1}+C_{2}\right)^{2} / g_{m} \ll 1$. Substituting Eq. (24) into Eq. (23) gives the lower limit to $g_{m}$ :

$$
10 \mathrm{mS}<g_{m}\left(1-\frac{n k}{1+\omega_{0}^{2} R_{L}^{2}\left(C_{1}+C_{2}\right)^{2}}\right) .
$$

In the frequency range from $\omega_{0}$ to $\omega_{1}$, the real and imaginary parts of $Y_{I N}$ reach the maximum values at $\omega_{1}$ and $\omega_{3}$, respectively:

$$
\begin{aligned}
& \operatorname{Re}\left[Y_{I N}(j \omega)\right]_{\max }=g_{m}+\frac{n^{2} k^{2}}{R_{L}}, \\
& \operatorname{Im}\left[Y_{I N}(j \omega)\right]_{\max }=\omega_{3} C_{p}-\frac{1}{\omega_{3} L_{p}}+\frac{n k}{2}\left(g_{m}+\frac{n k}{R_{L}}\right),
\end{aligned}
$$

where $\omega_{0}<\omega_{3}<\omega_{1}$ and $\omega_{3}<1 / \sqrt{L_{p} C_{p}}$ are assumed. Substituting Eqs. (26) and (27) into Eq. (22), we can have the worst case upper limit to $g_{m}$ and lower limit to $R_{L}$. For example, when $\operatorname{Im}\left[Y_{I N}(j \omega)\right]$ is negligible against $\operatorname{Re}\left[Y_{I N}(j \omega)\right]$ (around $\left.\omega_{1}\right)$, the following condition is derived from Eqs. (22) and (26):

$$
g_{m}+\frac{n^{2} k^{2}}{R_{L}}<38 \mathrm{mS} .
$$

An impedance matching procedure for the proposed LNA is as follows:

1. Select $g_{m}$ and $R_{L}$ to satisfy Eqs. (25) and (28)

2. Select $L_{p}$ such that $\omega_{0}$ equals the lower edge of the desired input band

3. Select $L_{1}$ such that $\omega_{4}$ equals the upper edge of the desired input band

Figure 7(c) shows the calculated $S_{11}$ of the LNAs with and without $L_{1}$, where $\omega_{0}$ and $\omega_{4}$ are set to approximately $3.1 \mathrm{GHz}$ and $10.6 \mathrm{GHz}$, respectively. A transconductance of $30 \mathrm{mS}$, a load resistance of $150 \Omega$, and other values shown in Fig. 7 result in $\operatorname{Re}\left[Y_{I N}(j \omega)\right]_{\max } \simeq 35 \mathrm{mS}$ and $\operatorname{Im}\left[Y_{I N}(j \omega)\right]_{\max } \simeq 10 \mathrm{mS}$, allowing $\left|S_{11}\right|<-10 \mathrm{~dB}$ from $\omega_{0}$ to $\omega_{1}$. Around $\omega_{4}$, the $\pi$ network including $L_{1}$ decreases $\operatorname{Re}\left[Y_{I N}(j \omega)\right]$ and $\operatorname{Im}\left[Y_{I N}(j \omega)\right]$ :

$$
\begin{aligned}
\operatorname{Re}\left[Y_{I N}\left(j \omega_{4}\right)\right]= & g_{m}-\frac{n k}{2 R_{L}}\left(\frac{\omega_{4} L_{1} C_{2} g_{m}}{C_{1}+C_{2}}-n k\right), \\
\operatorname{Im}\left[Y_{I N}\left(j \omega_{4}\right)\right]= & \omega_{4} C_{p}-\frac{1}{\omega_{4} L_{p}} \\
& -\frac{n k}{2 R_{L}}\left(\frac{\omega_{4} L_{1} C_{2} g_{m}}{C_{1}+C_{2}}+n k\right),
\end{aligned}
$$

providing $\left|S_{11}\right|<-10 \mathrm{~dB}$. Consequently, the input impedance matching is achieved from $\omega_{0}$ to $\omega_{4}$.

\subsection{Gain}

The transformer provides the positive feedback from node A to the input, as shown in Sect. 2.1. The transformer positive feedback reduces the gain $\left(S_{21}\right)$ bandwidth of the LNA. The $S_{21}$ of the LNA with output impedance matching is given by

$$
S_{21}=\frac{v_{\text {out }}}{v_{s} / 2}=\frac{2 v_{\text {in }}}{v_{s}} \frac{v_{\text {out }}}{v_{\text {in }}}=\frac{2}{1+R_{S} Y_{I N}} A_{v},
$$

where $v_{s}$ is the signal voltage and $A_{v}$, defined by $v_{\text {out }} / v_{\text {in }}$, is the voltage gain from the input to the output of the LNA, as shown in Fig. 2. Equation (31) shows that the frequency response of $A_{v}$ is shaped by that of $Y_{I N}$ (i.e., $S_{11}$ ), which results in that of $S_{21}$.

The frequency response of $A_{v}$ of the proposed LNA is similar to that of a CG LNA with a load resistor and output series inductor. The output network combining a shunt-peaking inductor with an output series inductor gives a larger bandwidth than the counterpart with either inductor, as explained in [23], [24]. However, the shunt-peaking inductor $L_{s}$ in the proposed LNA does not increase the bandwidth. The $A_{v}$ of the LNA is given by

$$
\begin{aligned}
& A_{v}(s)= \\
& \frac{g_{m} R_{L}\left(1+\frac{n k}{g_{m} R_{L}}+\frac{1-k^{2}}{m_{1}} \frac{s}{\omega_{c}}\right)}{1+\frac{s}{\omega_{c}}+\left(\frac{1-k^{2}}{m_{1}}+\frac{1-k_{c}}{m_{2}}\right) \frac{s^{2}}{\omega_{c}^{2}}+\frac{k_{c}\left(1-k_{c}\right.}{m_{2}} \frac{s^{3}}{\omega_{c}^{3}}+\frac{1-k^{2}}{m_{1}} \frac{k_{c}\left(1-k_{c}\right)}{m_{2}} \frac{s^{4}}{\omega_{c}^{4}}},
\end{aligned}
$$

where $k_{c}=C_{1} /\left(C_{1}+C_{2}\right), m_{1}=R_{L}^{2}\left(C_{1}+C_{2}\right) / L_{s}$, and $m_{2}=R_{L}^{2}\left(C_{1}+C_{2}\right) / L_{1}$ [24]. Substituting $k=0$ into Eq. (32) gives the $A_{v}$ of the CG LNA with both the shunt-peaking and output series inductors. Equation (32) shows that all $m_{1}$ are divided by a factor of $\left(1-k^{2}\right)$, i.e., $L_{s}$ is multiplied by a factor of $\left(1-k^{2}\right)$. This means that the effective $L_{s}$ in the proposed LNA becomes small, compared with the shunt-peaking inductor in the CG LNA, and then contributes less to bandwidth extension. The calculated $A_{v}$ with $k$ as a parameter is shown in Fig. 8, where $f_{c}=4.2 \mathrm{GHz}, k_{c}=0.4, m_{1}=1.6$, and $m_{2}=2.25$ originate from $C_{1}=100 \mathrm{fF}, C_{2}=150 \mathrm{fF}$, $L_{s}=3.5 \mathrm{nH}, L_{1}=2.5 \mathrm{nH}$, and $R_{L}=150 \Omega$. A very large peak (ripple) is found when $k=0$, because $L_{s}$ is larger than $L_{1}$, i.e., $m_{1}<m_{2}$ [23], [24]. Figure 8 shows that both the peak and bandwidth decrease as $k$ increases. Consequently, the bandwidth of the proposed LNA $(k \simeq 0.9)$ closely equals that of the CG LNA with only the output series inductor. A flat voltage gain of the CG LNA across the entire UWB frequency band can be obtained by selecting an appropriate value of $m_{2}$ (approximately 2), as discussed in [24].

An $S_{21}$ variation of the proposed LNA mainly originates from the characteristic of $Y_{I N}\left(S_{11}\right)$. As shown in Fig. 7(c), the input impedance matching condition improves around $\omega_{0}$ and $\omega_{4}$, but deteriorates around $\omega_{1}$. This means that an input signal of $\omega_{1}$ is less transferred to the input of 


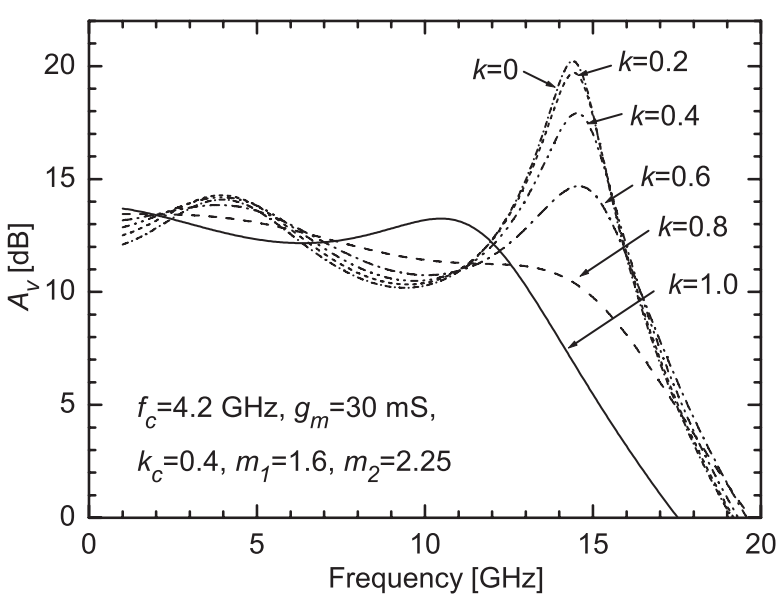

Fig. 8 Calculated $A_{v}$ of the proposed LNA with $k$ as a parameter.

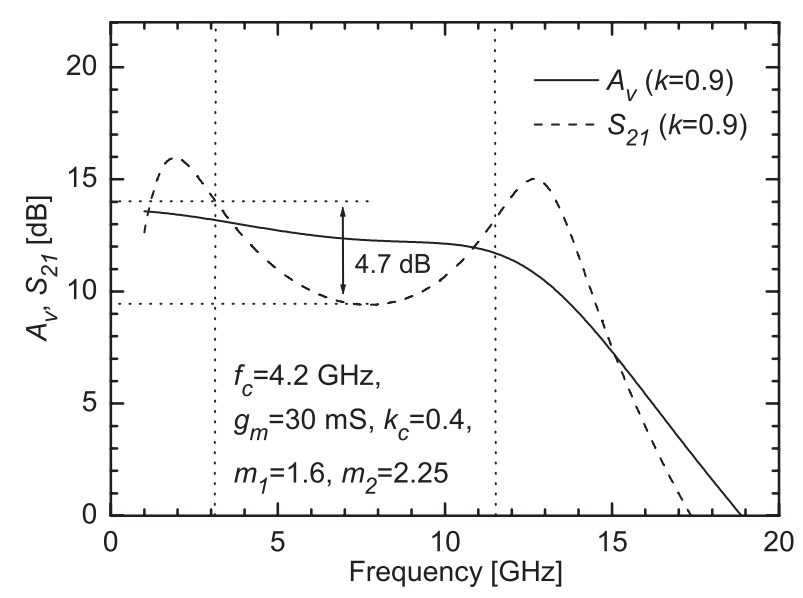

Fig. 9 Calculated $A_{v}$ and $S_{21}$ of the proposed LNA with $k=0.9$.

the LNA, compared to that of $\omega_{0}$ or $\omega_{4}$, which results in a reduction in the magnitude of $S_{21}$ around $\omega_{1}$. The difference between $S_{21}\left(j \omega_{0}\right)$ and $S_{21}\left(j \omega_{1}\right)$ can be approximated from Eq. (31):

$$
\begin{aligned}
\Delta S_{21} & =\frac{S_{21}\left(j \omega_{0}\right)}{S_{21}\left(j \omega_{1}\right)}=\frac{1+R_{S} Y_{I N}\left(j \omega_{1}\right)}{1+R_{s} Y_{I N}\left(j \omega_{0}\right)} \\
& \approx \frac{1+R_{s} \cdot \operatorname{Re}\left[Y_{I N}\left(j \omega_{1}\right)\right]}{1+R_{S} \cdot \operatorname{Re}\left[Y_{I N}\left(j \omega_{0}\right)\right]}
\end{aligned}
$$

where $A_{v}\left(j \omega_{0}\right)=A_{v}\left(j \omega_{1}\right)$ is assumed, the real parts of $Y_{I N}\left(j \omega_{0}\right)$ and $Y_{I N}\left(j \omega_{1}\right)$ are given by Eqs. (24) and (26), respectively, and the imaginary parts of $Y_{I N}\left(j \omega_{0}\right)$ and $Y_{I N}\left(j \omega_{1}\right)$ can be neglected, as shown in the previous subsection. Figure 9 shows the calculated $A_{v}$ and $S_{21}$ of the proposed LNA with $k=0.9$. Substituting the parameters shown in Fig. 9 into Eq. (33) gives $\Delta S_{21}=-3.6 \mathrm{~dB}$, while an $S_{21}$ variation of $-4.7 \mathrm{~dB}$ is seen in Fig. 9 , and then $-1.1 \mathrm{~dB}$ originates from the difference of $A_{v}$. The difference of $S_{21}$ can be reduced by decreasing $R_{L}$, as shown by Eqs. (24), (26), and (33). Moreover, using a common-source (CS) amplifier with a gain peak around $\omega_{1}$ as the second stage, we can obtain a flat gain.

\subsection{Stability}

The proposed LNA becomes potentially unstable, due to the transformer positive feedback. A linear two-port is unconditionally stable when it meets the following conditions [25]:

$$
\begin{aligned}
& \left|\Gamma_{S}\right|<1, \\
& \left|\Gamma_{L}\right|<1, \\
& \left|\Gamma_{I N}\right|<1, \\
& \left|\Gamma_{\text {OUT }}\right|<1,
\end{aligned}
$$

where $\Gamma_{S}, \Gamma_{I N}, \Gamma_{L}$, and $\Gamma_{\text {OUT }}$ represent the source, input, load, and output reflection coefficients, respectively. Equations (34)-(37) state that the real parts of the input and output impedances for passive source and load impedances must be positive [25]:

$$
\begin{aligned}
& \operatorname{Re}\left[Z_{I N}\right]>0, \\
& \operatorname{Re}\left[Z_{O U T}\right]>0 .
\end{aligned}
$$

In what follows, to simplify the expression of the output impedance of the LNA, we will verify whether the LNA without the output $\pi$ network $\left(C_{1}, L_{1}\right.$, and $\left.C_{2}\right)$ satisfies the above conditions or not.

First, the real part of the input admittance of the LNA can be derived from Eqs. (10)-(11) for $Z_{L}=\infty$ :

$$
\operatorname{Re}\left[Y_{I N}(j \omega)\right]=g_{m}(1-n k) .
$$

In the case of the proposed LNA, $n$ is selected to be one, shown in Sect. 3.1, and $k$ of the on-chip transformer is less than one [26]: $n k<1$. The requirement of Eq. (38) is thus satisfied. Next, the output impedance considering the left hand side of output node A (Fig. 2) is given by

$$
\begin{aligned}
& Z_{O U T, A}(j \omega)= \\
& \frac{\left(\frac{1}{R_{s}}+g_{m}+j \omega C_{p}\right)\left(R_{L}+j \omega\left(1-k^{2}\right) L_{s}\right)+n^{2}+\frac{R_{L}}{j \omega L_{p}}}{\frac{1}{R_{s}}+g_{m}(1-n k)+j \omega C_{p}+\frac{1}{j \omega L_{p}}} .
\end{aligned}
$$

Equation (41) indicates that the real part of $Z_{O U T, A}$ becomes a maximum around $\omega=1 / \sqrt{L_{p} C_{p}}$ and can be approximated by

$$
\begin{aligned}
& \operatorname{Re}\left[Z_{O U T, A}(j \omega)\right] \approx R_{L}, \\
& \operatorname{Re}\left[Z_{O U T, A}(j \omega)\right] \approx R_{L}+\frac{\left(1-k^{2}\right) L_{s}}{C_{p}}\left(\frac{1}{R_{s}}+g_{m}\right),
\end{aligned}
$$

at low and high frequencies (i.e., $\omega \ll 1 / \sqrt{L_{p} C_{p}}$ and $\omega \gg 1 / \sqrt{L_{p} C_{p}}$ ), respectively. The requirement of Eq. (39) is therefore satisfied.

The stability is also ensured through simulation. Figure 10 shows the simulated $K$ and $B_{1}$ of the proposed LNA, which are given by [25]

$$
K=\frac{1-\left|S_{11}\right|^{2}-\left|S_{22}\right|^{2}+|\Delta|^{2}}{2\left|S_{12} S_{21}\right|},
$$




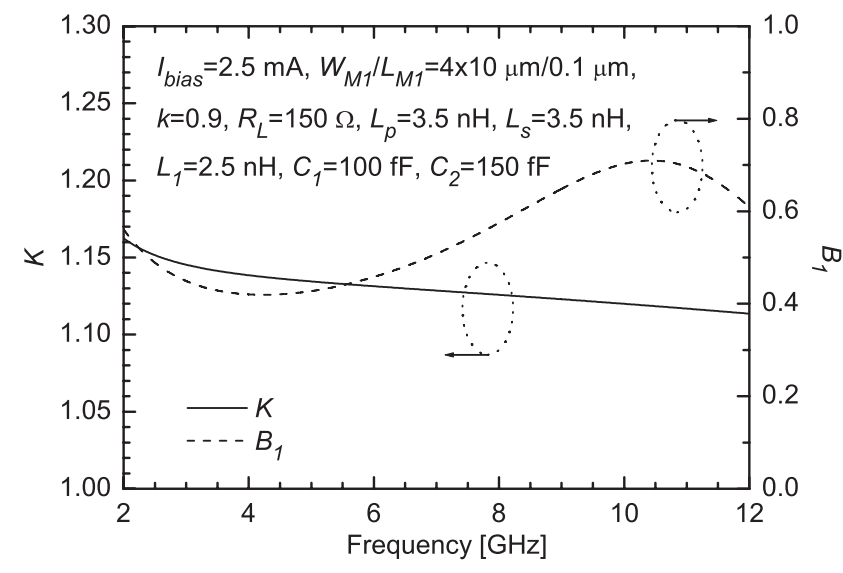

Fig. 10 Simulated $K$ and $B_{1}$ of the proposed LNA.

$$
B_{1}=1+\left|S_{11}\right|^{2}-\left|S_{22}\right|^{2}-|\Delta|^{2}
$$

respectively, where $\Delta=S_{11} S_{22}-S_{12} S_{21}$. The necessary and sufficient conditions for unconditional stability are $K>$ 1 and $B_{1}>0$ [25]. The simulations show that the LNA satisfies these conditions across the entire UWB frequency band.

\subsection{Group Delay}

A group delay variation is important for DS-UWB or pulsebased UWB systems. The group delay is the derivative of the phase of the signal transfer function $\left(S_{21}\right)$, and hence any resonance in the signal path contributes to the variation [27]. The critical resonances in the proposed LNA originate from the combinations of $L_{p}$ (transformer) and $C_{p}$ at the input, and $L_{1}, C_{1}$, and $C_{2}$ at the output, and these resonance frequencies, $\omega_{0}$ and $\omega_{2}$, are given by Eqs. (21) and (18), respectively. Pushing the resonance frequencies out of the desired frequency band (i.e., increasing $L_{p}$ or decreasing $L_{1}$ ) allows a small group delay variation. Figure 11 shows that the simulated group delays of the proposed LNA with $L_{p}$ and $L_{1}$ as a parameter. The group delay (for $L_{p}=3.0 \mathrm{nH}$ in Fig. 11(a)) dramatically changes around $3 \mathrm{GHz}\left(\omega_{0}\right)$ and $11 \mathrm{GHz}\left(\omega_{2}\right)$. The simulations also show that the variation can be reduced by increasing $L_{p}$ or decreasing $L_{1}$.

\section{Design}

By using a $90 \mathrm{~nm}$ CMOS process and device parameters, the proposed LNA is designed to satisfy the following typical specifications of the UWB LNA: $\left|S_{11}\right|<-10 \mathrm{~dB}, N F<$ $4 \mathrm{~dB}$, and $\left|S_{21}\right|>10 \mathrm{~dB}$ across the entire UWB frequency band $(3.1-10.6 \mathrm{GHz})$. Current consumption is set to $2.5 \mathrm{~mA}$ at a $1.0 \mathrm{~V}$ supply.

\subsection{Input Transistor and Load Resistor}

The transconductance $g_{m}$ and load resistance $R_{L}$ are determined by the input impedance matching conditions, given

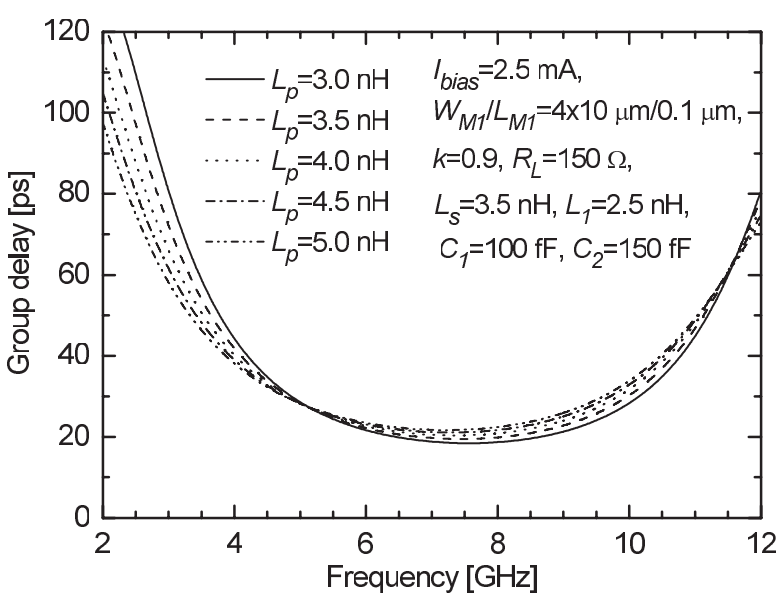

(a)

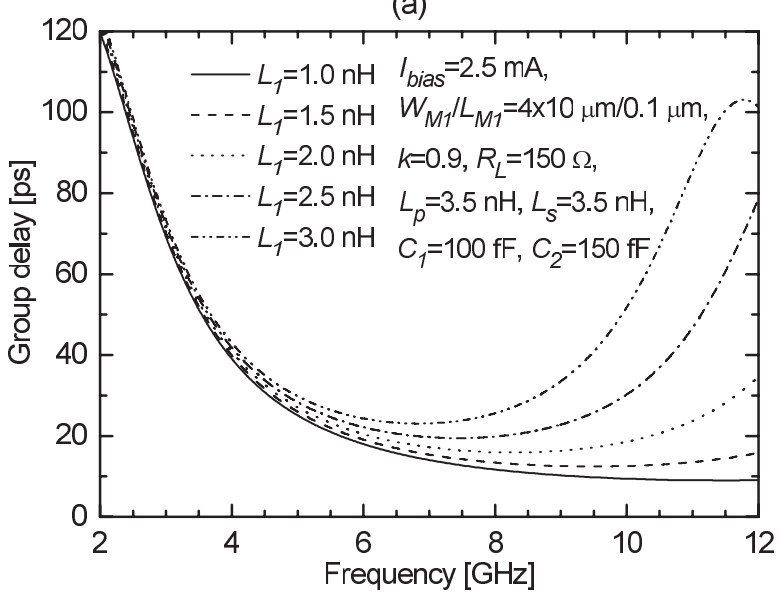

(b)

Fig. 11 Simulated group delays with (a) $L_{p}$ and (b) $L_{1}$ as a parameter.

by Eqs. (25) and (28), and the desired gain. A transconductance of $30 \mathrm{mS}$ and load resistance of $145 \Omega$ provide both $\left|S_{11}\right|<-10 \mathrm{~dB}$ and $A_{v} \simeq 14 \mathrm{~dB}^{\dagger}$ in the lower UWB band $(3.1-5 \mathrm{GHz})$. The load resistance includes the parasitic resistance of $L_{s}$. A bias current of $2.5 \mathrm{~mA}$ and $g_{m}$ of $30 \mathrm{mS}$ result in a gate width of $4 \times 10 \mu \mathrm{m}(10$ gate fingers, each with a unit of of $4 \mu \mathrm{m}$ width) and gate length of $100 \mathrm{~nm}$.

\subsection{Transformer}

The transformer adopts a stacked configuration in which $L_{p}$ is stacked on $L_{s}$. This configuration provides the largest coupling factor and a small area [26]. The large parasitic resistance of $L_{S}$, due to the lower thin metal layer, is not problematic, because it can be absorbed into $R_{L}$.

The parasitic capacitance between $L_{p}$ and $L_{s}, C_{c}$, has a relatively small effect on the LNA performance. This capacitance significantly affects the frequency response of a noninverting transformer [26]. Although the proposed LNA employs the noninverting stacked transformer, the signal current injected into $L_{s}$ by $M_{1}$ reduces the effect of $C_{c}$. Figure 12 shows the simulated $S_{11}$ and $N F$ of the LNA includ-

${ }^{\dagger}$ At low frequencies, $A_{v} \approx g_{m} R_{L}+n k=5.25$. 


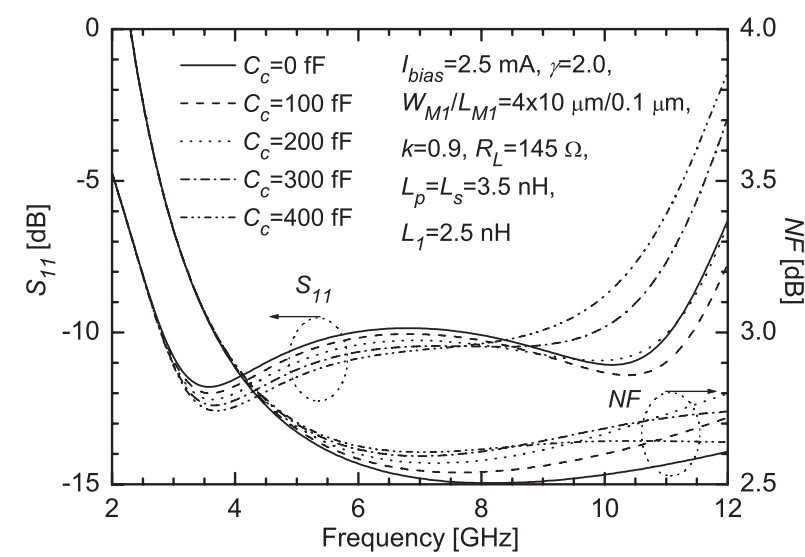

Fig. 12 Simulated $S_{11}$ and $N F$ of the LNA with $C_{c}$ as a parameter.

ing $C_{c}$. In the lower UWB band, $C_{c}$ slightly decreases the magnitude of the $S_{11}$ and has little impact on the $N F$; in the higher, a large $C_{c}$ decreases the input bandwidth and increases the $N F$ by up to $0.20 \mathrm{~dB}$. In the simulations, for wideband input impedance matching, $C_{c}$ must be less than $300 \mathrm{fF}$, which can be realized even with the stacked transformer.

The transformer is designed to achieve $\left|S_{11}\right|<-10 \mathrm{~dB}$ (of the LNA) in the lower UWB band and $N F<4.0 \mathrm{~dB}$ across the entire UWB band. Selecting $L_{p}$ such that $\omega_{0}$ equals approximately $3.1 \mathrm{GHz}$ allows the LNA to achieve $\left|S_{11}\right|<-10 \mathrm{~dB}$ in the lower band. A wide metal for realizing $L_{p}$ reduces the parasitic resistance; however, it leads to a large chip area and large parasitic capacitance. For $L_{p}$, we adopt a 3.5-turn square inductor with an outer diameter of $165 \mu \mathrm{m}$, metal width of $3 \mu \mathrm{m}$, and metal spacing of $2 \mu \mathrm{m}$. To achieve a turn ratio of one, $L_{s}$ is designed as follows: an outer diameter is $165 \mu \mathrm{m}$, a metal width $2 \mu \mathrm{m}$, and a metal spacing $3 \mu \mathrm{m}$. The metal thicknesses of $L_{p}$ (top pad metal) and $L_{s}$ (metal 6) are $1.9 \mu \mathrm{m}$ and $0.9 \mu \mathrm{m}$, respectively ${ }^{\dagger}$. The parasitic capacitance $C_{c}$ is reduced by offsetting the upper metal layer from the lower by short horizontal distance $(3 \mu \mathrm{m})$, which results in $C_{c} \simeq 240 \mathrm{fF}$. Three-dimensional (3-D) electromagnetic (EM) simulations by Ansoft HFSS showed $L_{p}=L_{s}=4.0 \mathrm{nH}$ and $k=0.9$ at low frequencies.

\subsection{Output Series Inductor}

The output series inductor $L_{1}$ is designed to set $\omega_{4}$ to the upper edge of the desired input band $(10.6 \mathrm{GHz})$. We use a relatively low $\mathrm{Q}$ inductor to reduce the chip area and parasitic capacitance, which reduces the gain bandwidth of the LNA. The outer diameter of $L_{1}$ is $140 \mu \mathrm{m}$, the metal width $3 \mu \mathrm{m}$, the metal spacing $2 \mu \mathrm{m}$, and the metal thickness $1.9 \mu \mathrm{m}$ (top pad metal $)^{\dagger}$. EM simulations showed that the inductance and maximum $Q$ were $3.2 \mathrm{nH}$ and 6.0 (at $5.0 \mathrm{GHz}$ ), respectively.

\section{Experimental Results and Discussion}

The designed LNA was fabricated in a $90 \mathrm{~nm}$ digital CMOS

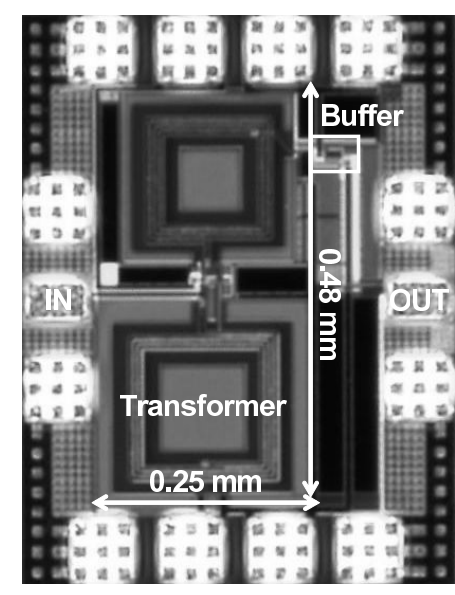

Fig. 13 Micrograph of the fabricated LNA.

process without metal-insulator-metal (MIM) capacitors. A micrograph of the fabricated LNA is shown in Fig. 13. The active chip area excluding pads was $0.48 \times 0.25 \mathrm{~mm}^{2}$. The input and output pads were not electrostatic-discharge (ESD) protected. Metal fills consisting of metal 1-6 layers were placed both inside and outside the fabricated transformer and inductor to meet metal density rules in the CMOS process. They were $1.5 \mu \mathrm{m}$ by $1.5 \mu \mathrm{m}$ squares with a spacing of $0.2 \mu \mathrm{m}$. The average horizontal distance between the metal fills and traces of the inductors was $15 \mu \mathrm{m}$. For measurements, a unity-gain CS amplifier with a $50 \Omega$ output resistor was used as a buffer. The S-parameters, noise, and linearity of the LNA were measured using on-wafer RF probes. The power consumption of the LNA and buffer were $2.5 \mathrm{~mW}$ and $4.0 \mathrm{~mW}$, respectively, at a supply voltage of $1.0 \mathrm{~V}$.

Figure 14 shows the measured and simulated $S_{11}$ and $S_{21}$ of the LNA. The LNA achieved $\left|S_{11}\right|<-10 \mathrm{~dB}$ and $\left|S_{21}\right|>9.3 \mathrm{~dB}$ across $3.1-10.6 \mathrm{GHz}$. The discrepancy between the measurements and simulations at frequencies above $4 \mathrm{GHz}$ is mainly attributed to insufficient accuracy in the simulations of the transformer and inductor used. The HFSS simulation models of the transformer and inductor included no metal fills to solve convergence problems and reduce the memory requirement. The metal fills increase the parasitic capacitances and resistances of the transformer and inductor [28]-[30], which results in the discrepancy.

Figure 15 shows the measured and simulated $S_{12}$ of the LNA with the buffer. The LNA achieved $\left|S_{12}\right|<-34 \mathrm{~dB}$ across $3.1-10.6 \mathrm{GHz}$. The measured $S_{12}$ of the stand-alone buffer (not shown) was less than $-24 \mathrm{~dB}$ over the same frequency range. Thus, the $S_{12}$ of the LNA without the buffer was less than $-10 \mathrm{~dB}$. The poor reverse isolation performance is due to the transformer, and an additional stage may be required to improve the isolation performance.

Figure 16 shows the measured and simulated group de-

†The inductors $L_{p}$ and $L_{1}$ in the previous work [17] were implemented by using metal 6 layers $(0.9 \mu \mathrm{m}$ thick $)$ and $L_{s}$ by using a metal 5 layer $(0.3 \mu \mathrm{m}$ thick). This leads to the differences between the simulations/measurements shown in [17] and in Sect. 5. 


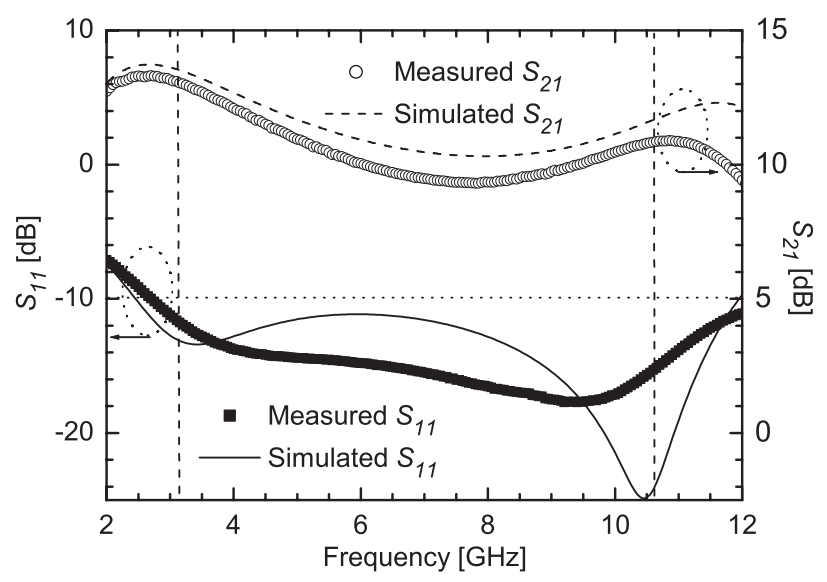

Fig. 14 Measured and simulated $S_{11}$ and $S_{21}$ of the LNA.

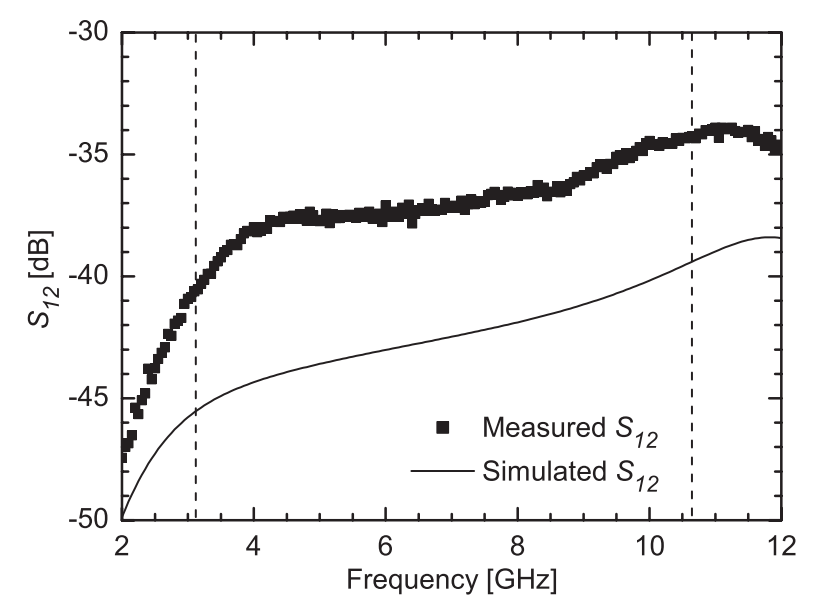

Fig. 15 Measured and simulated $S_{12}$ of the LNA.

lays. The group delay variation increased around the edge of the UWB frequency band, as analyzed in Sect. 3.5. A group delay variation of approximately $60 \mathrm{ps}$ was achieved for the entire band.

Figure 17 shows the measured and simulated $N F$ of the LNA. Note that these results included the noise of the output buffer, which increased the overall $N F$ by $0.8 \mathrm{~dB}$ for an LNA gain of $10 \mathrm{~dB}$ in simulation. The LNA with the buffer achieved an $N F$ of 3.8-4.4 $\mathrm{dB}^{\dagger}$ across the entire UWB band. This means that the proposed LNA with an additional CS amplifier like the buffer can achieve $N F<4.4 \mathrm{~dB}$. The difference between the measurements and simulations can be explained by the extra input-referred noise of the buffer, caused by the lower measured gain than the simulated one.

Figure 18 shows the measured output power of the fundamental tone and third-order intermodulation $\left(\mathrm{IM}_{3}\right)$ products for two tones $(3.000 \mathrm{GHz}$ and $3.001 \mathrm{GHz})$. The measured input third-order intercept point $\left(\mathrm{IIP}_{3}\right)$ and $1-\mathrm{dB}$ compression point (including the effect of the output buffer) were approximately $-9.3 \mathrm{dBm}$ and $-20 \mathrm{dBm}$, respectively. Figure 19 shows $I I P_{3}$ and $I I P_{2}$ measured by applying two tones $\left(f_{I 1}\right.$ and $\left.f_{I 2}\right)$ with $1-\mathrm{MHz}$ spacing. The measured frequency range of $3-6 \mathrm{GHz}$ was limited by a signal genera-

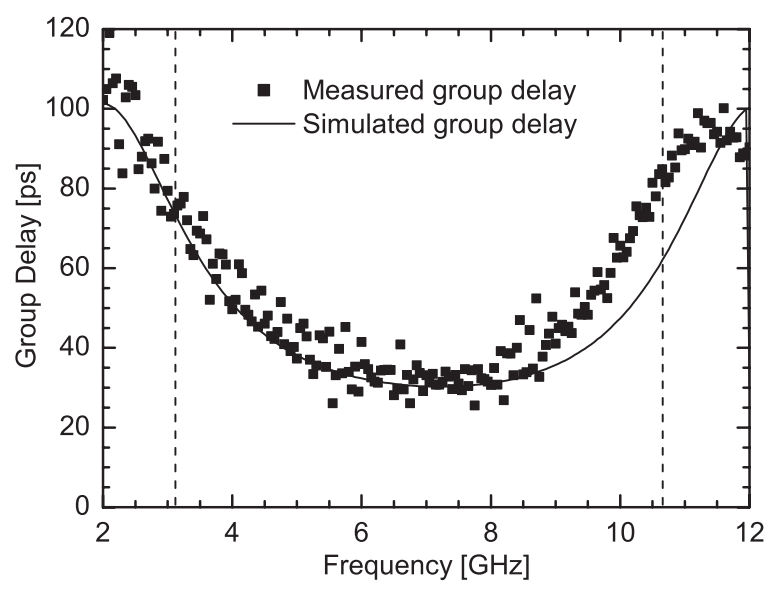

Fig. 16 Measured and simulated group delays of the LNA.

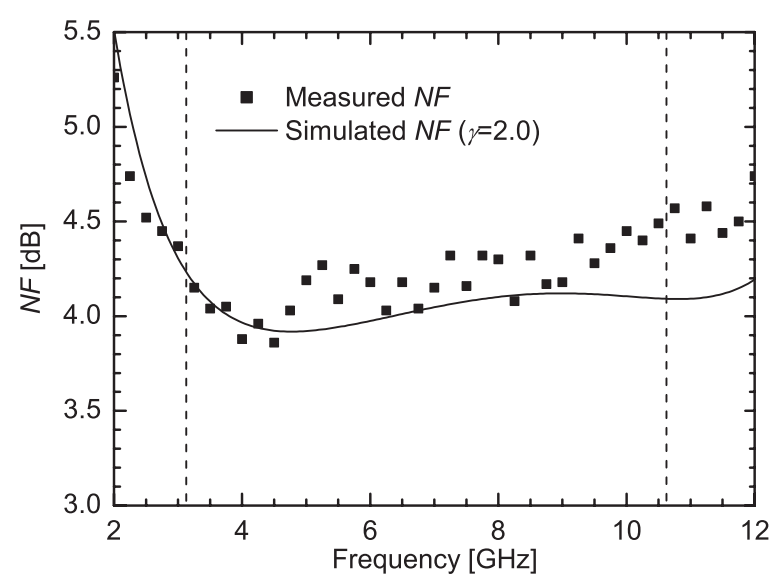

Fig. 17 Measured and simulated $N F$ of the LNA.

tor, and $\mathrm{IM}_{3}$ and $\mathrm{IM}_{2}$ products were measured at $2 f_{I 1}-f_{I 2}$ and $f_{I 1}+f_{I 2}^{\dagger \dagger}$, respectively. The LNA obtained $I I P_{3}>$ $-9.3 \mathrm{dBm}$ and $I I P_{2}>-6.3 \mathrm{dBm}$ in the frequency range.

Table 1 shows a summary of the LNA performance and a comparison with previously reported wideband CMOS LNAs. The proposed LNA achieved input impedance matching and comparable noise performance across the entire UWB band with the lowest reported power consumption and supply voltage. The LNA also consumed the smallest chip area among the 3.1-10.6 GHz LNAs employing inductors [10], [11], [16], [24].

An additional amplifier stage can allow the proposed LNA to achieve more and flatter gain across $3.1-10.6 \mathrm{GHz}$. A relatively low gain of the implemented LNA $(>9.3 \mathrm{~dB})$ leads to an increase in the overall $N F$ of the receiver. For instance, the $N F$ specification for RF receivers of the MB-

We found noise measurement errors in the previous work [17]. We overmeasured the loss of an input cable by approximately $1.0 \mathrm{~dB}$. This resulted in smaller measured $N F$ in [17] by $1.0 \mathrm{~dB}$.

† Due to the wideband characteristic of a UWB LNA, IM 2 products at $f_{I 1}+f_{I 2}$ generated by the LNA do not decrease and then affect the performance of a receiver. 
OFDM UWB system is less than $6.6 \mathrm{~dB}$ [31], [32]. A receiver employing the proposed LNA may have difficulty in satisfying such an $N F$ specification. A CS amplifier with a load inductor, shown in Fig. 20, improves the LNA gain, alleviating this problem. The CS amplifier is designed to have a gain peak around $8.0 \mathrm{GHz}$ and power consumption

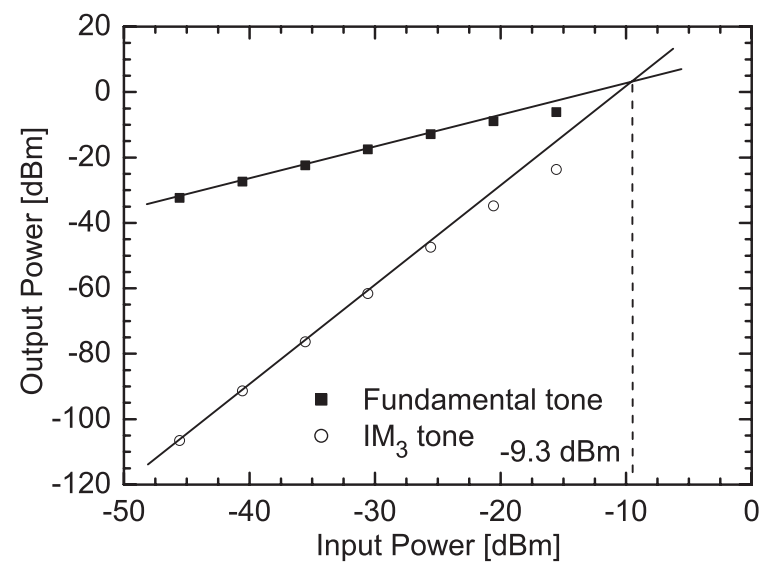

Fig. 18 Measured $I I P_{3}$ of the LNA at $3 \mathrm{GHz}$.

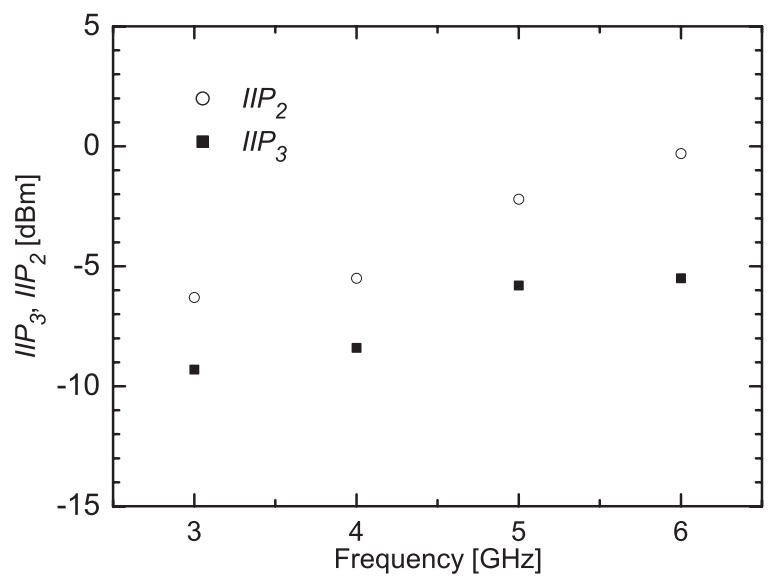

Fig. 19 Measured $I I P_{3}$ and $I I P_{2}$ of the LNA. of $2.0 \mathrm{~mW}$. The 2.6-nH inductor consists of stacked square spiral inductors implemented by the top pad metal and metal 6 layers, and occupies only $55 \times 55 \mu \mathrm{m}^{2}$. Figure 21 shows the simulated $S_{21}$ and $N F$ of the LNAs with and without the CS amplifier. The inductor was designed by using the EM simulator. The LNA with the CS amplifier achieved more and flatter gain $\left(\left|S_{21}\right|>20 \mathrm{~dB}\right)$ and the same noise performance as the LNA without the CS amplifier $(N F<4.3 \mathrm{~dB})$ across $3.1-10.6 \mathrm{GHz}$. The group delay variation (not shown) was reduced to approximately $20 \mathrm{ps}$. Considering the measurements of the fabricated LNA, we conclude that the proposed LNA with the CS amplifier can achieve $\left|S_{21}\right|>20 \mathrm{~dB}$ and $N F<4.4 \mathrm{~dB}$ across $3.1-10.6 \mathrm{GHz}$ with an additional power consumption of $2.0 \mathrm{~mW}$ and chip area of $55 \times 55 \mu \mathrm{m}^{2}$.

\section{Conclusion}

We have demonstrated a transformer noise-canceling UWB CMOS LNA with an output series inductor. The transformer partly cancels the noise of the common-gate transistor and load resistor, thereby improving the LNA noise performance. The output series inductor improves both the gain and input bandwidths. Circuit analysis showed

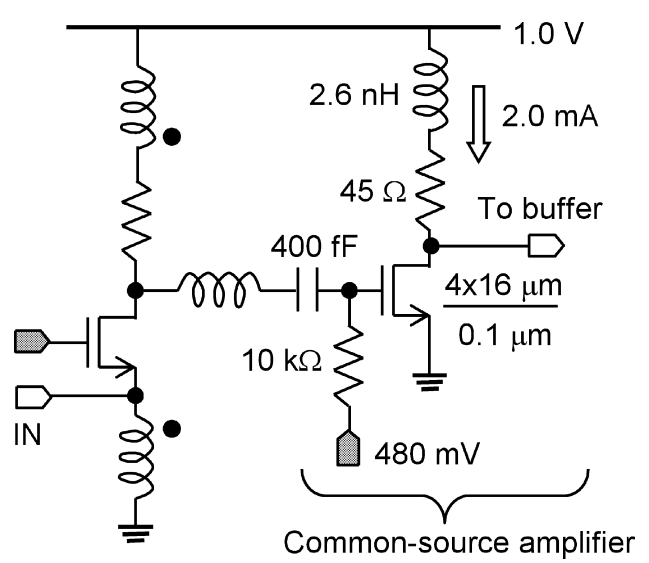

Fig. 20 Common-source amplifier with a load inductor

Table 1 Measured performance and comparison of wideband CMOS LNAs.

\begin{tabular}{c||c|c|c|c|c|c|c|c|c}
\hline \hline Reference & $\begin{array}{c}\text { CMOS } \\
\text { Technology }\end{array}$ & $\begin{array}{c}\mathrm{BW}^{*} \\
{[\mathrm{GHz}]}\end{array}$ & $\begin{array}{c}N F \\
{[\mathrm{~dB}]}\end{array}$ & $\begin{array}{c}S_{21} \\
{[\mathrm{~dB}]}\end{array}$ & $\begin{array}{c}I I P_{3} \\
{[\mathrm{dBm}]}\end{array}$ & $\begin{array}{c}\text { Supply } \\
{[\mathrm{V}]}\end{array}$ & $\begin{array}{c}\text { Power } \\
{[\mathrm{mW}]}\end{array}$ & $\begin{array}{c}\text { Area } \\
{\left[\mathrm{mm}^{2}\right]}\end{array}$ & Topology \\
\hline \hline This work & $90 \mathrm{~nm}$ & $2.8-12$ & $3.8-4.4^{\ddagger}$ & $9.3-13.1^{\ddagger}$ & -9.3 & 1.0 & 2.5 & 0.12 & Transformer noise-canceling CG \\
\hline$[4]$ & $90 \mathrm{~nm}$ & $0.5-6.2$ & $1.9-2.6$ & $25^{\circ}$ & -16 & 2.7 & 35.2 & 0.025 & Resistive feedback CS \\
\hline$[6]$ & $90 \mathrm{~nm}$ & $0.5-5.0$ & $2.3-2.6$ & $21-22^{\circ}$ & -8.8 & 1.8 & 12 & 0.012 & Resistive feedback CS \\
\hline$[7]$ & $90 \mathrm{~nm}$ & $0-6$ & $3.4-4.3$ & $12.5-15.3$ & N/A & 1.0 & 3.4 & 0.0017 & Common-drain feedback CS \\
\hline$[8]$ & $130 \mathrm{~nm}$ & $1-7.4$ & $>2.4$ & $15-17^{\circ}$ & -4.1 & 1.4 & 25 & 0.019 & Common-drain feedback CS \\
\hline$[10]$ & $130 \mathrm{~nm}$ & $3.1-10.6$ & $2.1-2.9$ & $13.7-16.5$ & -8.5 & 1.2 & 9.0 & 0.40 & Reactive feedback CS \\
\hline$[11]$ & $180 \mathrm{~nm}$ & $0-14.1$ & $4.5-5.1^{\ddagger}$ & $12.0-13.7^{\ddagger}$ & -6.2 & 1.8 & 20 & 0.50 & Noise-canceling CG using CS \\
\hline$[13]$ & $65 \mathrm{~nm}$ & $0.2-6.2$ & $2.8-4.2$ & $9.9-15.6^{\circ}$ & 0 & 1.2 & 14 & 0.009 & Noise-canceling CG using CS \\
\hline$[14]$ & $130 \mathrm{~nm}$ & $3.7-8.8$ & $3.6-4.5$ & $8.1-11$ & -7.2 & 1.5 & 19 & 0.05 & Noise-canceling CS \\
\hline$[16]$ & $180 \mathrm{~nm}$ & $1-11$ & $2.9-3.0$ & $8-9$ & -3.55 & 1.8 & 21.6 & 0.20 & Distributed \\
\hline$[24]$ & $180 \mathrm{~nm}$ & $2.6-10.7$ & $4.4-5.3^{\ddagger}$ & $6.0-8.5^{\ddagger}$ & 7.4 & 1.8 & 4.5 & 0.40 & Capacitor cross-coupled CG ${ }^{\star}$ \\
\hline \hline
\end{tabular}

${ }^{*}$ Input bandwidth ${ }^{\dagger}$ Without buffers ${ }^{\ddagger} 3.1-10.6 \mathrm{GHz}$ frequency range ${ }^{\triangleright}$ Voltage gain ${ }^{\star}$ Input-output differential topology 


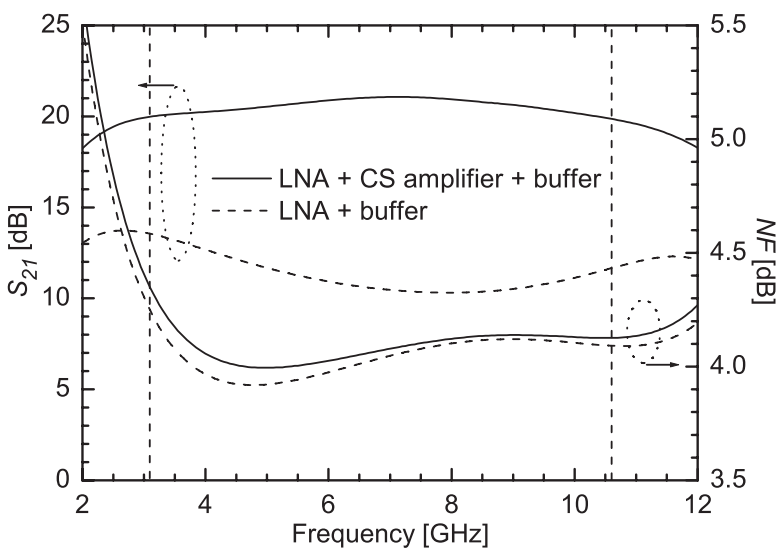

Fig. 21 Simulated $S_{21}$ and $N F$ of the LNAs with and without the common-source amplifier.

that the best turn ratio for the noise performance is one and input impedance matching depends not only on the common-gate transistor but also on the load resistor. The LNA designed for UWB applications was fabricated in a $90 \mathrm{~nm}$ digital CMOS process. The fabricated LNA occupied $0.12 \mathrm{~mm}^{2}$, and achieved $\left|S_{11}\right|<-10 \mathrm{~dB}, N F<4.4 \mathrm{~dB}$, and $\left|S_{21}\right|>9.3 \mathrm{~dB}$ across $3.1-10.6 \mathrm{GHz}$, while consuming $2.5 \mathrm{~mW}$ from a $1.0 \mathrm{~V}$ supply. The proposed topology is the most suitable for low-power and low-voltage UWB CMOS LNAs.

\section{Acknowledgment}

The chip in this study was fabricated through the chip fabrication program of the VLSI Design and Education Center (VDEC), the University of Tokyo, in collaboration with Semiconductor Technology Academic Research Center (STARC), Fujitsu Limited, Matsushita Electric Industrial Company Limited., NEC Electronics Corporation, Renesas Technology Corporation, and Toshiba Corporation. In addition, this study was financially supported by a grant to the Osaka University Global COE Program, "Center for Electronic Devices Innovation" from the Ministry of Education, Culture, Sports, Science and Technology of Japan, and Initiatives for Attractive Education in Graduate Schools from Japan Society for the Promotion of Science (JSPS).

\section{References}

[1] MultiBand OFDM Alliance SIG, "Multiband OFDM physical layer proposal for IEEE 802.15 task group 3a," Sept. 2004.

[2] R. Fisher, R. Kohno, H. Ogawa, H. Zhang, K. Takizawa, M. McLaughlin, and M. Welborn, "DS-UWB physical layer submission to 802.15 task group 3a," Sept. 2005.

[3] A. Bevilacqua and A.M. Niknejad, "An ultrawideband CMOS lownoise amplifier for 3.1-10.6 GHz wireless receivers," IEEE J. SolidState Circuits, vol.39, no.12, pp.2259-2268, Dec. 2004.

[4] J.H.C. Zhan and S.S. Taylor, "A $5 \mathrm{GHz}$ resistive-feedback CMOS LNA for low-cost multi-standard applications," IEEE Int. SolidState Circuits Conf. Dig. Tech. Papers, pp.200-201, San Francisco, CA, Feb. 2006

[5] B.G. Perumana, J.H.C. Zhan, S.S. Taylor, and J. Laskar, "A 12 mW,
7.5 GHz bandwidth, inductor-less CMOS LNA for low-power, lowcost, multi-standard receivers," IEEE Radio Frequency Integrated Circuits Symp. Dig. Papers, pp.57-60, Honolulu, HI, June 2007.

[6] B.G. Perumana, J.H.C. Zhan, S.S. Taylor, B.R. Carlton, and J. Laskar, "Resistive-feedback CMOS low-noise amplifiers for multiband applications," IEEE Trans. Microw. Theory Tech., vol.56, no.5, pp.1218-1225, May 2008.

[7] J. Borremans, P. Wambacq, and D. Linten, "An ESD-protected DCto-6 GHz $9.7 \mathrm{~mW}$ LNA in $90 \mathrm{~nm}$ digital CMOS," IEEE Int. SolidState Circuits Conf. Dig. Tech. Papers, pp.422-423, San Francisco, CA, Feb. 2007

[8] R. Ramzan, S. Andersson, J. Dabrowski, and C. Svensson, "A 1.4 V $25 \mathrm{~mW}$ inductorless wideband LNA in $0.13 \mu \mathrm{m}$ CMOS," IEEE Int. Solid-State Circuits Conf. Dig. Tech. Papers, pp.424-425, San Francisco, CA, Feb. 2007.

[9] M.T. Reiha, J.R. Long, and J.J. Pekarik, "A $1.2 \mathrm{~V}$ reactivefeedback $3.1-10.6 \mathrm{GHz}$ ultrawideband low-noise amplifier in $0.13 \mu \mathrm{m}$ CMOS," IEEE Radio Frequency Integrated Circuits Symp. Dig. Papers, pp.41-44, San Francisco, CA, June 2006.

[10] M.T. Reiha and J.R. Long, "A $1.2 \mathrm{~V}$ reactive-feedback 3.1-10.6 GHz low-noise amplifier in $0.13 \mu \mathrm{m}$ CMOS," IEEE J. Solid-State Circuits, vol.42, no.5, pp.1023-1033, May 2007.

[11] C.F. Liao and S.I. Liu, "A broadband noise-canceling CMOS LNA for 3.1-10.6-GHz UWB receivers," IEEE J. Solid-State Circuits, vol.42, no.2, pp.329-339, Feb. 2007.

[12] S. Chehrazi, A. Mirzaei, R. Bagheri, and A.A. Abidi, "A $6.5 \mathrm{GHz}$ wideband CMOS low noise amplifier for muti-band use," Proc. IEEE Custom Integrated Circuits Conf., pp.801-804, San Francisco, CA, Sept. 2005.

[13] S.C. Blaakmeer, E.A. Klumperink, B. Nauta, and D.M. Leenaertsr, "An inductorless wideband balun-LNA in $65 \mathrm{~nm}$ CMOS with balanced output," Proc. IEEE European Solid-State Circuits Conf., pp.364-367, Munich, Germany, Sept. 2007.

[14] Q. Li and Y.P. Zhang, "A 1.5-V 2-9.6-GHz inductorless low-noise amplifier in 0.13- $\mu \mathrm{m}$ CMOS," IEEE Trans. Microw. Theory Tech., vol.55, no.10, pp.2015-2023, Oct. 2007.

[15] F. Zhang and P.R. Kinget, "Low-power programmable gain CMOS distributed LNA," IEEE J. Solid-State Circuits, vol.41, no.6, pp.1333-1343, June 2006.

[16] P. Heydari, "Design and analysis of a performance-optimized CMOS UWB distributed LNA," IEEE J. Solid-State Circuits, vol.42, no.9, pp.1892-1905, Sept. 2007.

[17] T. Kihara, T. Matsuoka, and K. Taniguchi, "A 1.0 V, $2.5 \mathrm{~mW}$, transformer noise-canceling UWB CMOS LNA," IEEE Radio Frequency Integrated Circuits Symp. Dig. Papers, pp.493-496, Atlanta, GA, June 2008.

[18] D.J. Allstot, X. Li, and S. Shekhar, "Design considerations for CMOS low-noise amplifiers," IEEE Radio Frequency Integrated Circuits Symp. Dig. Papers, pp.97-100, Fort Worth, TX, June 2004.

[19] A. Liscidini, C. Ghezzi, E. Depaoli, G. Albasini, I. Bietti, and R. Castello, "Common gate transformer feedback LNA in a high IIP3 current mode RF CMOS front-end," Proc. IEEE Custom Integrated Circuits Conf., pp.25-28, San Francisco, CA, July 2006.

[20] P.P. Jindal, "Compact noise models for MOSFETs," IEEE Trans. Electron Devices, vol.53, no.9, pp.2051-2061, Sept. 2006.

[21] Y. Kiyota, C.H. Chen, T. Kubodera, A. Nakamura, K. Takeshita, and M.J. Deen, "A new approach of high frequency noise modeling for 70-nm NMOS transistors by accurate noise source extraction,” IEEE Radio Frequency Integrated Circuits Symp. Dig. Papers, pp.635638, Honolulu, HI, June 2007.

[22] J. Jussila and P. Sivonen, "A 1.2-V highly linear balanced noisecancelling LNA in $0.13-\mu \mathrm{m}$ CMOS," IEEE J. Solid-State Circuits, vol.43, no.3, pp.579-587, March 2008

[23] S. Galal and B. Razavi, "40-Gb/s amplifier and ESD protection circuit in 0.18- $\mu \mathrm{m}$ CMOS technology," IEEE J. Solid-State Circuits, vol.39, no.12, pp.2389-2396, Dec. 2004.

[24] S. Shekhar, J.S. Walling, and D.J. Allstot, "Bandwidth extension 
techniques for CMOS amplifiers," IEEE J. Solid-State Circuits, vol.41, no.11, pp.2424-2439, Nov. 2006.

[25] G. Gonzalez, Microwave Transistor Amplifiers, 2 ed., Prentice Hall, Upper Saddle River, NJ, 1997.

[26] J.R. Long, "Monolithic transformers for silicon RF IC design," IEEE J. Solid-State Circuits, vol.35, no.9, pp.1368-1381, Sept. 2000.

[27] Y. Park, C.H. Lee, J.D. Cressler, and J. Laskar, "The analysis of UWB SiGe HBT LNA for its noise, linearity, and minimum group delay variation," IEEE Trans. Microw. Theory Tech., vol.54, no.4, pp.1687-1697, April 2006.

[28] J.H. Chang, Y.S. Youn, H.K. Yu, and C.K. Kim, "Effects of dummy patterns and substrate on spiral inductors forsub-micron RF ICs," IEEE Radio Frequency Integrated Circuits Symp. Dig. Papers, pp.419-422, Seattle, WA, June 2002.

[29] F. Zhang and P.R. Kinget, "Design of components and circuits underneath integrated inductors," IEEE J. Solid-State Circuits, vol.41, no.10, pp.2265-2271, Oct. 2006.

[30] L. Nan, K. Mouthaan, Y.Z. Xiong, J. Shi, S.C. Rustagi, and B.L. Ooi, "Experimental characterization of the effect of metal dummy fills on spiral inductors," IEEE Radio Frequency Integrated Circuits Symp. Dig. Papers, pp.307-310, Honolulu, HI, June 2007.

[31] A. Batra, J. Balakrishnan, G.R. Aiello, J.R. Foerster, and A. Dabak, "Design of a multiband OFDM system for realistic UWB channel environments," IEEE Trans. Microw. Theory Tech., vol.52, no.9, pp.2123-2138, Sept. 2004.

[32] R. Roovers, D.M.W. Leenaerts, J. Bergervoet, K.S. Harish, R.C.H. van de Beek, G. van der Weide, H. Waite, Y. Zhang, S. Aggarwal, and C. Razzell, "An interference-robust receiver for ultra-wideband radio in SiGe BiCMOS technology," IEEE J. Solid-State Circuits, vol.40, no.12, pp.2563-2572, Dec. 2005.

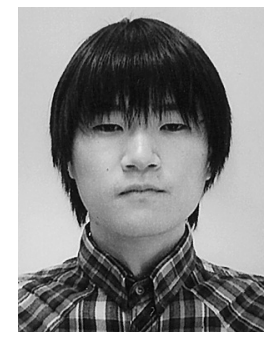

Takao Kihara received the B.S., M.S., and $\mathrm{Ph} . \mathrm{D}$. degrees in electronic engineering from Osaka University, Osaka, Japan, in 2005, 2006 , and 2009 , respectively. His current research interests include CMOS RF transceivers.

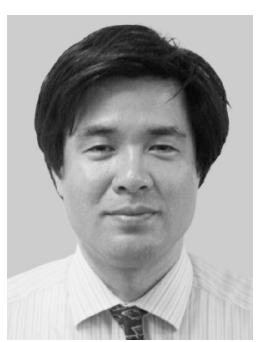

Toshimasa Matsuoka received the B.S., M.S., and Ph.D. degrees in electronic engineering from Osaka University, Osaka, Japan, in 1989, 1991, and 1996, respectively. During 1991-1998, he worked for the Central Research Laboratories, Sharp Corporation, Nara, Japan, where he was engaged in the research and development of deep submicron CMOS devices and ultra thin gate oxides. Since 1999, he has been working for Osaka University, where he is Associate Professor now. His current research includes MOS device modeling and CMOS RF circuits. Dr. Matsuoka is a member of the Japan Society of Applied Physics (JSAP), the Institute of Electrical Engineers of Japan (IEEJ), and the Institute of Electrical and Electronic Engineers (IEEE).

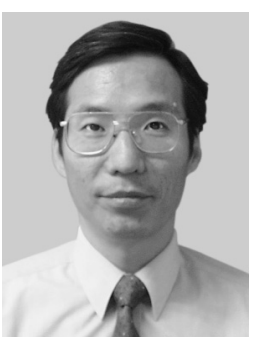

Kenji Taniguchi received the B.S., M.S., and Ph.D. degrees from Osaka University, Osaka, Japan, in 1971, 1973, and 1986, respectively. From 1973 to 1986, he worked for Toshiba Research and Development Center, Kawasaki, Japan where he was engaged in process modeling and the design of MOS LSI fabrication technology. He was a Visiting Scientist at Massachusetts Institute of Technology, Cambridge, from July 1982 to November 1983 Presently, he is a Professor of Electrical, Electronic and Information Engineering at Osaka University. His current research interests are analog circuits, radio frequency circuits, device physics and process technology. Prof. Taniguchi is a member of the Japan Society of Applied Physics (JSAP), the Institute of Electrical Engineers of Japan (IEEJ), and the Institute of Electrical and Electronic Engineers (IEEE). 